Supporting information

\title{
Discovery of highly potent inhibitors targeting the predominant drug-resistant S31N mutant of the influenza A virus M2 proton channel
}

\author{
Fang Li, Chunlong Ma, William F. DeGrado, Jun Wang \\ junwang@pharmacy.arizona.edu
}

\section{Table of Contents}

Characterizations of intermediates

Table S1

Characterizations of final products

Table S2

\section{Chemistry}

All chemicals were purchased from commercial vendors and used without further purification

unless otherwise noted. ${ }^{1} \mathrm{H}$ and ${ }^{13} \mathrm{C}$ NMR spectra were recorded on a Bruker-300 NMR spectrometer. Chemical shifts are reported in parts per million referenced with respect to residual solvent $\left(\mathrm{CD}_{3} \mathrm{OD}\right) 3.31 \mathrm{ppm}$ and $\left(\mathrm{DMSO}-d_{6}\right) 2.50 \mathrm{ppm}$ or from internal standard tetramethylsilane (TMS) $0.00 \mathrm{ppm}$. The following abbreviations were used in reporting spectra: (s) singlet, (d) doublet, (t) triplet, (q) quartet, (m) multiplet, (dd) doublet of doublets, (ddd) doublet of doublet of doublets. All reactions were carried out under $\mathrm{N}_{2}$ atmosphere, unless otherwise stated. HPLC grade solvents were used for all reactions. Flash column chromatography was performed using silica gel (230-400 mesh, Merck). Low-resolution mass 
spectra were obtained using an ESI technique on a 3200 Q Trap LC/MS/MS system (Applied Biosystems). The purity was assessed using Shimadzu LC-MS with Waters XTerra MS C18 column (part \# 186000538), $50 \times 2.1 \mathrm{~mm}$, at a flow rate of $0.3 \mathrm{ml} / \mathrm{min} ; \lambda=250$ and $220 \mathrm{~nm}$; mobile phase A, $0.1 \%$ formic acid in $\mathrm{H}_{2} \mathrm{O}$, and mobile phase $\mathrm{B}$, $0.1 \%$ formic in $60 \%$ isopropanol, $30 \% \mathrm{CH}_{3} \mathrm{CN}$ and $9.9 \% \mathrm{H}_{2} \mathrm{O}$. The purified fractions were lyophilized. All compounds submitted for testing in TEVC assay and plaque reduction assay were confirmed to be $>95.0 \%$ purity by LC-MS traces. All compounds were characterized by proton NMR; selected compounds were also characterized by carbon NMR.

\section{Method I: General procedure of reductive amination (Scheme 1A):}

Adamantane (1 equiv) and aldehyde (1 equiv) were mixed with $2 \mathrm{~mL}$ titanium (IV) isopropoxide. The resulting slurry was heated to $100{ }^{\circ} \mathrm{C}$ and stirred overnight. Then the solution was cooled down to $0{ }^{\circ} \mathrm{C}$ in ice bath, $\mathrm{CH}_{3} \mathrm{OH}$ was added, and $\mathrm{NaBH}_{4}$ (4 equiv) was added portionwise in 10 min. The solution was warmed to room temperature and stirred for 4 hrs. The reaction was quenched with $1 \mathrm{M} \mathrm{NaOH}$ and filtered through Celite. The filtrate was concentrated under reduced pressure and purified by silica gel flash column chromatography (5-10\% $\mathrm{CH}_{3} \mathrm{OH} / \mathrm{CH}_{2} \mathrm{Cl}_{2}$ ) to give the final product.

\section{Method II: General procedure of alkylations (Scheme 1B and Scheme 2C):}

The chloride/bromide ( 1 equiv) and amantadine (1.5 equiv) were dissolved in isopropanol; CsI (0.1 equiv) and triethyl amine ( 2 equiv) were then added. The reaction mixture was heated to reflux overnight. The solvent was removed under reduced pressure, and the resulting residue was extracted with ethyl acetate and water. The organic layer was separated, dried over anhydrous 
magnesium sulfate, filtered, and concentrated under reduced pressure. The mixture was then purified by silica gel flash column chromatography $\left(5-10 \% \mathrm{CH}_{3} \mathrm{OH} / \mathrm{CH}_{2} \mathrm{Cl}_{2}\right)$ to give the final product.

\section{Method III: General procedure of ester reduction by $\mathrm{NaBH}_{4}\left(\mathrm{Scheme}_{2 \mathrm{~A}}\right)$ :}

Ester ( 1 equiv) was dissolved in methanol and cooled down to $0{ }^{\circ} \mathrm{C} . \mathrm{NaBH}_{4}$ (4 equiv) was added in small portions to the solution over $10 \mathrm{~min}$. The mixture was warmed slowly to room temperature and stirred for four hours. Diluted $\mathrm{HCl}$ was added and the organic solvent was removed under reduced pressure. The resulting aqueous layer was extracted with ethyl acetate $(3 \times)$, and the organic layers were combined and dried over $\mathrm{MgSO}_{4}$, and the solvent was removed under reduced pressure. This hydroxyl intermediate was used for the next step without further purification.

Method IV: General procedure of bromination by $\mathrm{PPh}_{3}$ and $\mathrm{CBr}_{4}\left(\mathrm{Scheme}_{2} \mathrm{~A}\right)$ :

Hydroxyl intermediate (1 equiv) was dissolved in DCM, and the resulting solution was cooled down to $0{ }^{\circ} \mathrm{C} . \mathrm{CBr}_{4}\left(1.5\right.$ equiv) and $\mathrm{PPh}_{3}(1.5$ equiv) were added sequentially. The solution was stirred at $0{ }^{\circ} \mathrm{C}$ for $20 \mathrm{~min}$ and gradually warmed up to room temperature. The solvent was removed under reduced pressure, and the residue was purified by flash column chromatography (20-100\% hexane/DCM) to give the desired bromide intermediate.

\section{Method V: General procedure for the synthesis of isoxazole (Scheme 2A):}

To a stirred solution of dimethyl oxalate (1.1 equiv) and aryl methyl ketone ( 1 equiv) in toluene was added a solution of potassium tert-butoxide in THF (1.2 equiv) dropwise. The resulting 
solution was stirred at room temperature overnight. The reaction was quenched with $1 \mathrm{~N} \mathrm{HCl}$ and extracted with ethyl acetate $(3 \mathrm{X})$. The combined organic layers were dried over $\mathrm{MgSO}_{4}$, filtered and concentrated under reduced pressure. The crude product was dissolved in methanol and hydroxylamine hydrochloride (1.5 equiv) was added. The solution was heated to $50{ }^{\circ} \mathrm{C}$ for $6 \mathrm{hrs}$. The solvent was removed under reduced pressure and the resulting isoxazole ester was purified by flash column chromatography. The ester was subsequently reduced by $\mathrm{NaBH}_{4}$ (Method III), converted to bromide (Method IV), and underwent alkylation (Method II) to give the final product.

\section{Method VI: Procedure for the synthesis of 1,2,4-oxadiazole (Scheme 2B):}

Carboxylic acid (1 equiv) and 2-chloro-N-hydroxyethanimidamide (1 equiv) were dissolved in DMF. DIEA (2 equiv) and HCTU (1 equiv) were added. The resulting solution was stirred at room temperature for 2 hrs. Water was added and the solution was extracted with DCM (3X). The combined organic layers were dried over $\mathrm{MgSO}_{4}$, filtered and concentrated under reduced pressure. The residue was dissolved in DMF and heated to $135^{\circ} \mathrm{C}$ overnight. Water was added and the solution was extracted with DCM (3X). The combined organic layers were dried over $\mathrm{MgSO}_{4}$, filtered and concentrated under reduced pressure. The residue was purified by flash column chromatography to give the 1,2,4-oxadiazole chloride. The chloride was subsequently underwent alkylation (Method II) to give the final product.

\section{Method VII: Procedure for the synthesis of 1,3,4-oxadiazole (Scheme 3):}

2-thiophenecarboxylic acid hydrazide (1 equiv) and $\mathrm{Et}_{3} \mathrm{~N}$ (2 equiv) were dissolved in $\mathrm{CH}_{2} \mathrm{Cl}_{2}$ at $0{ }^{\circ} \mathrm{C}$, methyl oxalate chloride ( 1 equiv) was added dropwise. The reaction mixture was warmed slowly to room temperature and stirred for 6 hours. TsCl (1 equiv) was added and stirred 
overnight. The mixture was diluted with $\mathrm{CH}_{2} \mathrm{Cl}_{2}$ and was washed with water and saturated brine. The organic layer was dried over $\mathrm{MgSO}_{4}$ and the solvent was removed under reduced pressure. The crude produce was purified by flash column chromatography to give the ester intermediate. The ester was subsequently reduced by $\mathrm{NaBH}_{4}$ (Method III), converted to bromide (Method IV), and underwent alkylation (Method II) to give the final product.

\section{Method VIII: Procedure for the synthesis of 1,3,4-thiadiazole (Scheme 3):}

2-thiophenecarboxylic acid hydrazide (1 equiv) and $\mathrm{Et}_{3} \mathrm{~N}$ (2 equiv) were dissolved in $\mathrm{CH}_{2} \mathrm{Cl}_{2}$ at $0{ }^{\circ} \mathrm{C}$, methyl oxalate chloride ( 1 equiv) was added dropwise. The reaction mixture was warmed slowly to room temperature and stirred overnight. The solvent was removed under reduced pressure. The residue was dissolved in toluene and treated with Lawesson's reagent (1.5 equiv) and heated at $110^{\circ} \mathrm{C}$ for 6 hours. The mixture was cooled down to room temperatureand ethyl acetate was added. The slurry was washed with water, saturated $\mathrm{NaHCO}_{3}$, and saturated brine. The organic layer was dried over $\mathrm{MgSO}_{4}$, and the solvent was removed under reduced pressure. The crude produce was purified by flash column chromatography to give the ester intermediate. The ester was subsequently reduced by $\mathrm{NaBH}_{4}$ (Method III), converted to bromide (Method IV), and underwent alkylation (Method II) to give the final product.

\section{Synthesis procedures for Scheme 4}

Tert-butyl N-(4-oxoadamantan-1-yl)carbamate (28)

To a mixture of diphenylphosphorylazide (DPPA) (1.3 mL, $6.1 \mathrm{mmol}, 1.2$ equiv) and triethylamine $(0.85 \mathrm{~mL}, 6.1 \mathrm{mmol}, 1.2$ equiv) in $10 \mathrm{~mL}$ dichloromethane was added a solution of 4-oxoadamantane-1-carboxylic acid (1 g, $5.1 \mathrm{mmol}, 1$ equiv) at ambient temperature. The 
reaction was stirred for $2 \mathrm{~h}$. An additional $10 \mathrm{~mL}$ dichloromethane and $10 \mathrm{~mL} \mathrm{H}_{2} \mathrm{O}$ were added to the reaction mixture, and the organic layer was separated and washed sequentially with $\mathrm{H}_{2} \mathrm{O}$, saturated $\mathrm{NaHCO}_{3}(10 \mathrm{~mL})$, and brine and dried over anhydrous magnesium sulfate. Solvent was removed by rotary evaporation and $t-\mathrm{BuOH}(25 \mathrm{ml})$ was added. The solution was heated to reflux under $\mathrm{N}_{2}$ atmosphere for $5 \mathrm{~h}$. Then the mixture was concentrated under reduced pressure and purified by silica gel flash column chromatography $\left(0-10 \% \mathrm{CH}_{3} \mathrm{OH} / \mathrm{CH}_{2} \mathrm{Cl}_{2}\right)$ to give 28 as white solid (1.27 g, $4.8 \mathrm{mmol}, 78 \%)^{1}$

5-aminoadamantan-2-one (29)

A solution of $28(0.8 \mathrm{~g}, 3.0 \mathrm{mmol})$ in hexafluoro-2-propanol (HFIP, $15 \mathrm{~mL})$ was heated at $150{ }^{\circ} \mathrm{C}$ by Biotage Initiator Classic microwave synthesizer for $60 \mathrm{~min}$. Then the mixture was concentrated under reduced pressure, and the crude was used in the next reaction without purification. $^{2}$

5-(\{[5-(thiophen-2-yl)-1,2-oxazol-3-yl]methyl $\}$ amino)adamantan-2-one (30a)

Compound 30a was synthesized by alkylation following the general procedure as described in Method II. The final product was purified by silica gel flash column chromatography (5-10\% $\mathrm{CH}_{3} \mathrm{OH} / \mathrm{CH}_{2} \mathrm{Cl}_{2}$ ) to give the final product.

\section{5-(\{[5-(thiophen-2-yl)-1,2-oxazol-3-yl]methyl $\}$ amino)adamantan-2-ol (30b)}

To a mixture of 30a (50 mg, $0.15 \mathrm{mmol}, 1$ equiv) in dry methanol $(3 \mathrm{~mL})$ at $0{ }^{\circ} \mathrm{C}$ was added sodium borohydride (11.5 mg, $0.3 \mathrm{mmol}, 2$ equiv). The mixture was stirred at $0{ }^{\circ} \mathrm{C}$ for $30 \mathrm{~min}$ then warmed back to ambient temperature in $60 \mathrm{~min}$ and stirred for $120 \mathrm{~min}$. Saturated 
ammonium chloride solution $(5 \mathrm{~mL})$ was added at $0{ }^{\circ} \mathrm{C}$, and the mixture was extracted by dichloromethane $(3 \times 5 \mathrm{~mL})$. The organic layer was separated, dried over anhydrous magnesium sulfate, filtered, and concentrated under reduced pressure. The mixture was then purified by silica gel flash column chromatography $\left(0-10 \% \mathrm{CH}_{3} \mathrm{OH} / \mathrm{CH}_{2} \mathrm{Cl}_{2}\right)$ to give the final product as a pale yellow solid (33 mg, $0.1 \mathrm{mmol}, 67 \%$ ).

2-methyl-5-(\{[5-(thiophen-2-yl)-1,2-oxazol-3-yl]methyl $\}$ amino)adamantan-2-ol (30c)

To a mixture of 30a ( $89 \mathrm{mg}, 0.27 \mathrm{mmol}, 1$ equiv) in dry tetrahydrofuran $(3 \mathrm{~mL})$ at $0{ }^{\circ} \mathrm{C}$ was added methyl magnesium chloride ( $270 \mu \mathrm{L}, 3 \mathrm{M}, 3$ equiv). The mixture was stirred at $0{ }^{\circ} \mathrm{C}$ for 5 $\mathrm{h}$ then quenched by saturated ammonium chloride solution $(5 \mathrm{~mL})$. The mixture was extracted by dichloromethane $(3 \times 5 \mathrm{~mL})$. The organic layer was separated, dried over anhydrous magnesium sulfate, filtered, and concentrated under reduced pressure. The mixture was then purified by silica gel flash column chromatography $\left(0-10 \% \mathrm{CH}_{3} \mathrm{OH} / \mathrm{CH}_{2} \mathrm{Cl}_{2}\right)$ to give the final product as a yellow solid (51 mg, $0.15 \mathrm{mmol}, 55 \%)$.

$\mathrm{N}$-\{[5-(thiophen-2-yl)-1,2-oxazol-3-yl]methyl\} spiro[adamantane-2,2'-oxirane]-7-amine (30d)

A solution of the ylide was prepared by adding sodium hydride $(17 \mathrm{mg}, 0.43 \mathrm{mmol}, 4.2$ tetrohydrofuran) to a solution of trimethyloxosulfonium iodide ( $94 \mathrm{mg}, 0.43 \mathrm{mmol}, 4.2$ equiv) in dimethylsulfoxide $(1 \mathrm{ml})$ at ${ }^{\circ} \mathrm{C}$ under nitrogen. After stirring for $60 \mathrm{~min}$, the ylide was added dropwise to the pre-cooled $\left(0{ }^{\circ} \mathrm{C}\right)$ solution of 30a $(34 \mathrm{mg}, 0.1 \mathrm{mmol}, 1$ equiv) in dimethylsulfoxide and tetrahydrofuran $(1 \mathrm{ml}, 1: 1)$. The mixture was stirred at $0{ }^{\circ} \mathrm{C}$ for $60 \mathrm{~min}$ then warmed back to ambient temperature and stirred for $120 \mathrm{~min}$. Saturated ammonium chloride solution $(2 \mathrm{~mL})$ was added at $0{ }^{\circ} \mathrm{C}$ and the mixture was extracted by dichloromethane $(3 \times 2 \mathrm{~mL})$. 
The organic layer was separated, dried over anhydrous magnesium sulfate, filtered, and concentrated under reduced pressure. The mixture was then purified by silica gel flash column chromatography $\left(0-10 \% \mathrm{CH}_{3} \mathrm{OH} / \mathrm{CH}_{2} \mathrm{Cl}_{2}\right)$ to give the final product as a pale yellow solid (24 $\mathrm{mg}$, $0.072 \mathrm{mmol}, 72 \%)^{3}$

$\mathrm{N}-\{[5$-(thiophen-2-yl)-1,2-oxazol-3-yl $]$ methyl $\}$-4-oxatricyclo[4.3.1.1 $\left.{ }^{3},{ }^{8}\right]$ undecan-1-amine (30e) To a solution of 28 (310 mg, $1.2 \mathrm{mmol}, 1$ equiv) in dichloromethane (20 mL) were added 3chloroperbenzoic acid (m-CPBA, $420 \mathrm{mg}, 1.9 \mathrm{mmol}, 1.6$ equiv) and sodium bicarbonate (160 mg, 1.9 mmol, 1.6 equiv) at $0{ }^{\circ} \mathrm{C}$, then warmed back to ambient temperature and stirred for $60 \mathrm{~min}$. The mixture was then diluted with saturated sodium bisulfate solution $(10 \mathrm{~mL})$ and extracted with dichloromethane $(3 \times 10 \mathrm{~mL})$. The organic layer was separated, dried over anhydrous magnesium sulfate, filtered, and concentrated under reduced pressure. The crude was used in the next reaction without purification. ${ }^{4}$

To a solution of this crude in chloroform $(15 \mathrm{~mL})$ were added indium tribromide $(42 \mathrm{mg}, 0.12$ mmol, 0.1 equiv) and triethyl silane (4.6 mL, $28.8 \mathrm{mmol}, 24$ equiv); the mixture was heated at $60{ }^{\circ} \mathrm{C}$ for $60 \mathrm{~min}$ and then cooled to ambient temperature. The reaction was diluted with water $(10 \mathrm{~mL})$ and extracted by dichloromethane $(3 \times 10 \mathrm{~mL})$. The organic layer was separated, dried over anhydrous magnesium sulfate, filtered, and concentrated under reduced pressure. The crude was used in the next reaction without purification. ${ }^{4}$

A solution of this crude in hexafluoro-2-propanol(HFIP, $15 \mathrm{~mL}$ ) was heated at $150{ }^{\circ} \mathrm{C}$ by Biotage Initiator Classic microwave synthesizer for $60 \mathrm{~min}$. Then the mixture was concentrated 
under reduced pressure, and the crude was directly used in Method II reaction and provided 30e as a yellow solid (107 $\mathrm{mg}, 0.32 \mathrm{mmol}, 27 \%$ in four steps).

3-(\{[5-(thiophen-2-yl)-1,2-oxazol-3-yl]methyl $\}$ amino)adamantan-1-ol $\quad$ (30f) and $3-(\{[5-(3-$ methoxythiophen-2-yl)-1,2-oxazol-3yl]methyl\}amino)adamantan-1-ol (30g) Compounds 30f and 30g were synthesized by alkylation following the general procedure as described in Method II.

5-(\{[5-(thiophen-2-yl)-1,2-oxazol-3-yl]methyl $\}$ amino)adamantane-1,3-diol (30h)

To a mixture of potassium hydroxide ( $26 \mathrm{mg}, 0.424 \mathrm{mmol}, 1$ equiv) and potassium permanganate (74 mg, $0.466 \mathrm{mmol}, 1.1 \mathrm{mmol})$ in $\mathrm{H}_{2} \mathrm{O}(10 \mathrm{~mL})$ at $50{ }^{\circ} \mathrm{C}$ was added 3-biomo-adamantane-1carboxylic acid (34, $110 \mathrm{mg}, 0.424 \mathrm{mmol}, 1$ equiv). The reaction mixture was heated at $98{ }^{\circ} \mathrm{C}$ for $18 \mathrm{~h}$. After cooling to ambient temperature, the mixture was acidified by the addition of $6 \mathrm{~N}$ aqueous $\mathrm{HCl}$, then saturated aqueous sodium bisulfite was added. The mixture was extracted with 2-propanol/ $\mathrm{CH}_{2} \mathrm{CI}_{2}(1: 3)$. The organic extract was concentrated to afford 35 and used in the next reaction without purification. ${ }^{5}$

To a mixture of diphenylphosphorylazide (DPPA) $(0.11 \mathrm{~mL}, 0.51 \mathrm{mmol}, 1.2$ equiv) and triethylamine ( $0.07 \mathrm{~mL}, 0.51 \mathrm{mmol}, 1.2$ equiv) in $3 \mathrm{~mL}$ dichloromethane was added a solution of 35 crude ( 0.424 mmol, 1 equiv) in $1 \mathrm{ml}$ dichloromethane at ambient temperature. The reaction was stirred for $2 \mathrm{~h}$. An additional $5 \mathrm{~mL}$ dichloromethane and $5 \mathrm{ml} \mathrm{H}_{2} \mathrm{O}$ were added to the reaction mixture, the organic layer was separated and washed sequentially with $\mathrm{H}_{2} \mathrm{O}(5 \mathrm{~mL})$, saturated $\mathrm{NaHCO}_{3}(5 \mathrm{~mL})$, and brine $(5 \mathrm{~mL})$, and dried over $\mathrm{MgSO}_{4}$. Solvent was removed by 
rotary evaporation and $t$ - $\mathrm{BuOH}(5 \mathrm{ml})$ was added. The solution was heated to reflux under $\mathrm{N}_{2}$ atmosphere for $5 \mathrm{~h}$. Then the mixture was concentrated under reduced pressure, dissolved in hexafluoro-2-propanol (HFIP, $5 \mathrm{~mL}$ ), and heated at $150{ }^{\circ} \mathrm{C}$ by Biotage Initiator Classic microwave synthesizer for $60 \mathrm{~min}$. Then the mixture was concentrated under reduced pressure then submitted for Method II coupling and provided 30h as a pale yellow solid (25 mg, 0.072 mmol, $17 \%$ in five steps).

The following compounds were synthesized according to Method V. (isoxazole synthesis):

8a, 14d, 14g, 14k, 14n, 14o, 14p, 14r, 14s.

The following compounds were synthesized according to Method I (reductive amination):

14f, 14i, 14j, 18e, $26 \mathrm{e}$.

The following compounds were synthesized according to Method II (alkylation):

8b, 8d, 14b, 14e, 14h, 14m, 14t, 18b, 18g, 18h, 18j, 18l, 20a, 20b, 20c, 26c, 26d, 26h, $26 \mathrm{i}$.

The following compounds were synthesized according to Method VI:

$18 \mathrm{~m}$.

The following compounds was synthesized according to Method VII:

26a.

The following compound was synthesized according to Method VIII: 
$26 b$.

Table S1: Characterizations of intermediates

\begin{tabular}{|c|c|}
\hline & $\begin{array}{l}\text { methyl 5-[4-(methylsulfanyl)phenyl]-1,2-oxazole-3-carboxylate (Yield: } \\
70 \%) .{ }^{1} \mathrm{HNMR}\left(300 \mathrm{MHz}, \mathrm{CDCl}_{3}\right): \delta 7.70(\mathrm{~d}, J=8.64 \mathrm{~Hz}, 2 \mathrm{H}), 7.30 \\
(\mathrm{~d}, J=8.64 \mathrm{~Hz}, 2 \mathrm{H}), 6.87(\mathrm{~s}, 1 \mathrm{H}), 4.00(\mathrm{~s}, 3 \mathrm{H}), 2.52(\mathrm{~s}, 3 \mathrm{H}) .{ }^{13} \mathrm{CNMR}: \\
\delta 171.60,160.59,156.78,142.97,126.29,126.15,122.99,99.48 \text {, } \\
53.02,15.18 .\end{array}$ \\
\hline & $\begin{array}{l}\text { 3-(bromomethyl)-5-[4-(methylsulfanyl)phenyl]-1,2-oxazole. } \quad \text { (Yield: } \\
68 \%) .{ }^{1} \mathrm{HNMR}\left(300 \mathrm{MHz}, \mathrm{CDCl}_{3}\right): \delta 7.68(\mathrm{~d}, J=8.55 \mathrm{~Hz}, 2 \mathrm{H}), 7.30 \\
(\mathrm{~d}, J=8.55 \mathrm{~Hz}, 2 \mathrm{H}), 6.57(\mathrm{~s}, 1 \mathrm{H}), 4.47(\mathrm{~s}, 2 \mathrm{H}), 2.54(\mathrm{~s}, 3 \mathrm{H}) .\end{array}$ \\
\hline & $\begin{array}{l}\text { methyl 5-(3-methoxyphenyl)-1,2-oxazole-3-carboxylate. (Yield: 82 \%). } \\
{ }^{1} \mathrm{HNMR}\left(300 \mathrm{MHz}, \mathrm{CDCl}_{3}\right): \delta \text { 7.43-7.35 (m, 3H), 7.05-7.01 (m, 1H), } \\
\text { 6.94-6.91 (m, 1H), } 4.02(\mathrm{~s}, 3 \mathrm{H}), 3.89(\mathrm{~s}, 3 \mathrm{H}) .\end{array}$ \\
\hline $\mathrm{Br}$ & $\begin{array}{l}\text { 3-(bromomethyl)-5-(3-methoxyphenyl)-1,2-oxazole. (Yield: } 70 \%) \text {. } \\
{ }^{1} \mathrm{HNMR}\left(300 \mathrm{MHz}, \mathrm{CDCl}_{3}\right): \delta 7.41-7.27(\mathrm{~m}, 3 \mathrm{H}), 7.02-6.98(\mathrm{~m}, 1 \mathrm{H}) \text {, } \\
6.61(\mathrm{~s}, 1 \mathrm{H}), 4.47(\mathrm{~s}, 2 \mathrm{H}), 3.87(\mathrm{~s}, 3 \mathrm{H}) .{ }^{13} \mathrm{CNMR}\left(75 \mathrm{MHz}, \mathrm{CDCl}_{3}\right): \delta \\
170.86,161.59,160.11,130.30,128.31,118.48,116.61,111.05,99.87 \text {, } \\
55.57,20.83 .\end{array}$ \\
\hline $\bar{F}$ & $\begin{array}{l}\text { methyl 5-(2,6-difluorophenyl)-1,2-oxazole-3-carboxylate. (Yield: } \\
65 \%) .{ }^{1} \mathrm{HNMR}\left(300 \mathrm{MHz}^{\mathrm{CDCl}}{ }_{3}\right): \delta 750-7.43(\mathrm{~m}, 1 \mathrm{H}), 7.11-7.04(\mathrm{~m} \text {, } \\
3 \mathrm{H}), 4.01(\mathrm{~s}, 3 \mathrm{H}) .\end{array}$ \\
\hline
\end{tabular}




\begin{tabular}{|c|c|}
\hline & $\begin{array}{l}\text { 3-(bromomethyl)-5-(3-methoxyphenyl)-1,2-oxazole. (Yield: } 77 \% \text { ). } \\
{ }^{1} \mathrm{HNMR}\left(300 \mathrm{MHz}, \mathrm{CDCl}_{3}\right): \delta 7.46-7.40(\mathrm{~m}, 1 \mathrm{H}), 7.10-7.06(\mathrm{~m}, 2 \mathrm{H}) \text {, } \\
6.82-6.80(\mathrm{~m}, 1 \mathrm{H}), 4.51(\mathrm{~s}, 2 \mathrm{H}) .\end{array}$ \\
\hline & $\begin{array}{l}\text { methyl 5-(2-bromophenyl)-1,2-oxazole-3-carboxylate. (Yield: } 84 \%) \text {. } \\
{ }^{1} \mathrm{HNMR}\left(300 \mathrm{MHz} \mathrm{CDCl}_{3}\right): \delta 7.89-7.86(\mathrm{~m}, 1 \mathrm{H}), 7.73-7.70(\mathrm{~m}, 1 \mathrm{H}) \text {, } \\
7.47-7.42(\mathrm{~m}, 1 \mathrm{H}), 7.34(\mathrm{~s}, 1 \mathrm{H}), 7.34-7.24(\mathrm{~m}, 1 \mathrm{H}), 4.00(\mathrm{~s}, 3 \mathrm{H}) \text {. } \\
{ }^{13} \mathrm{CNMR}\left(75 \mathrm{MHz} \mathrm{CDCl}_{3}\right): \delta 170.02,160.87,156.91,134.88,132.18 \text {, } \\
130.67,128.37,128.02,121.80,150.17,53.49 .\end{array}$ \\
\hline & $\begin{array}{l}\text { 3-(bromomethyl)-5-(2-bromophenyl)-1,2-oxazole. (Yield: } 83 \%) \text {. } \\
{ }^{1} \mathrm{HNMR}\left(300 \mathrm{MHz}, \mathrm{CDCl}_{3}\right): \delta 7.88-7.85(\mathrm{~m}, 1 \mathrm{H}), 7.77-7.70(\mathrm{~m}, 1 \mathrm{H}) \text {, } \\
7.47-7.44(\mathrm{~m}, 1 \mathrm{H}), 7.33-7.30(\mathrm{~m}, 1 \mathrm{H}), 7.07(\mathrm{~s}, 1 \mathrm{H}), 4.50(\mathrm{~s}, 2 \mathrm{H}) \text {. } \\
{ }^{13} \mathrm{CNMR}\left(75 \mathrm{MHz}, \mathrm{CDCl}_{3}\right): \delta 168.61,161.31,134.46,131.49,130.24 \text {, } \\
128.13,127.95,121.33,104.49,20.84 .\end{array}$ \\
\hline & $\begin{array}{l}\text { methyl 5-[2-(methylsulfanyl)phenyl]-1,2-oxazole-3-carboxylate. } \\
\text { (Yield: } 81 \%) .{ }^{1} \mathrm{HNMR}\left(300 \mathrm{MHz}, \mathrm{CDCl}_{3}\right): \delta 7.83-7.80(\mathrm{~m}, 1 \mathrm{H}), 7.45- \\
7.42(\mathrm{~m}, 1 \mathrm{H}), 7.40-7.35(\mathrm{~m}, 1 \mathrm{H}), 7.28-7.23(\mathrm{~m}, 2 \mathrm{H}), 4.00(\mathrm{~s}, 3 \mathrm{H}), 2.51 \\
(\mathrm{~s}, 3 \mathrm{H}) .{ }^{13} \mathrm{CNMR}\left(75 \mathrm{MHz}, \mathrm{CDCl}_{3}\right): \delta 169.66,160.66,156.58,138.02 \text {, } \\
130.98,129.24,126.38,125.25,125.20,104.60,53.05,16.17 .\end{array}$ \\
\hline $\mathrm{Br}$ & $\begin{array}{l}\text { 3-(bromomethyl)-5-[2-(methylsulfanyl)phenyl]-1,2-oxazole. (Yield: } \\
77 \%) .{ }^{1} \mathrm{HNMR}\left(300 \mathrm{MHz} \mathrm{CDCl}_{3}\right): \delta 7.82-7.79(\mathrm{~m}, 1 \mathrm{H}), 7.45-7.42(\mathrm{~m} \text {, } \\
1 \mathrm{H}), 7.35-7.29(\mathrm{~m}, 1 \mathrm{H}), 7.28-7.23(\mathrm{~m}, 1 \mathrm{H}), 6.96(\mathrm{~s}, 1 \mathrm{H}), 4.50(\mathrm{~s}, 2 \mathrm{H}) \text {, } \\
2.53(\mathrm{~s}, 3 \mathrm{H}) .{ }^{13} \mathrm{CNMR}\left(75 \mathrm{MHz}, \mathrm{CDCl}_{3}\right): \delta 168.67,161.33,137.77 \text {, } \\
130.65,129.18,136.26,125.75,125.21,104.27,20.99,16.19 .\end{array}$ \\
\hline
\end{tabular}




\begin{tabular}{|c|c|}
\hline & $\begin{array}{l}\text { methyl 5-(3,5-difluorophenyl)-1,2-oxazole-3-carboxylate. (Yield: } \\
65 \%) .{ }^{1} \mathrm{HNMR}\left(300 \mathrm{MHz}, \mathrm{CDCl}_{3}\right): \delta 7.36-7.34(\mathrm{~m}, 2 \mathrm{H}), 6.98-6.92(\mathrm{~m}, \\
\text { 2H), } 4.02(\mathrm{~s}, 3 \mathrm{H}) .\end{array}$ \\
\hline & $\begin{array}{l}\text { [5-(3,5-difluorophenyl)-1,2-oxazol-3-yl]methanol. }(\text { Yield: } 92 \%) \\
{ }^{1} \mathrm{HNMR}\left(300 \mathrm{MHz}, \mathrm{CDCl}_{3}\right): \delta \text { 7.27-7.24 (m, 2H), 6.91-6.87 (m, 1H), } \\
6.63(\mathrm{~s}, 1 \mathrm{H}), 4.81(\mathrm{~s}, 2 \mathrm{H}), 2.93(\mathrm{br} \mathrm{s}, 1 \mathrm{H}) .\end{array}$ \\
\hline & $\begin{array}{l}\text { methyl 5-cyclobutyl-1,2-oxazole-3-carboxylate. (Yield: } 61 \%) \\
{ }^{1} \mathrm{HNMR}\left(300 \mathrm{MHz}, \mathrm{CDCl}_{3}\right): \delta 6.43(\mathrm{~s}, 1 \mathrm{H}), 3.71(\mathrm{~s}, 3 \mathrm{H}), 3.68-3.65(\mathrm{~m}, \\
1 \mathrm{H}), 2.46-2.40(\mathrm{~m}, 4 \mathrm{H}), 2.26-2.04(\mathrm{~m}, 2 \mathrm{H}) .\end{array}$ \\
\hline & $\begin{array}{l}\text { 3-(bromomethyl)-5-cyclobutyl-1,2-oxazole. (Yield: } 75 \% \text { ). }{ }^{1} \mathrm{HNMR} \\
\left(300 \mathrm{MHz}, \mathrm{CDCl}_{3}\right): \delta 6.09(\mathrm{~s}, 1 \mathrm{H}), 4.39(\mathrm{~s}, 2 \mathrm{H}), 3.65-3.59 \text { (m, 1H), } \\
\text { 2.40-2.25 (m, 4H), 2.11-1.98 (m, 2H). }\end{array}$ \\
\hline & $\begin{array}{l}\text { methyl 5-(thiophen-2-yl)-1,3,4-thiadiazole-2-carboxylate. (Yield: } \\
75 \%) .{ }^{1} \mathrm{HNMR}\left(300 \mathrm{MHz}, \mathrm{CDCl}_{3}\right): \delta 7.73-7.71(\mathrm{~s}, 1 \mathrm{H}), 7.64-7.62(\mathrm{~s}, \\
1 \mathrm{H}), 7.23-7.20(\mathrm{~s}, 1 \mathrm{H}), 4.11(\mathrm{~s}, 3 \mathrm{H}) .{ }^{13} \mathrm{CNMR}\left(75 \mathrm{MHz}, \mathrm{CDCl}_{3}\right): \delta \\
166.49,159.43,158.45,131.61,131.25,131.19,128.54,53.91 . \text { EI-MS: } \\
m / z\left(\mathrm{M}+\mathrm{H}^{+}\right): 227.3 \text { (calculated), } 227.4 \text { (found). }\end{array}$ \\
\hline $\mathrm{Br}$ & 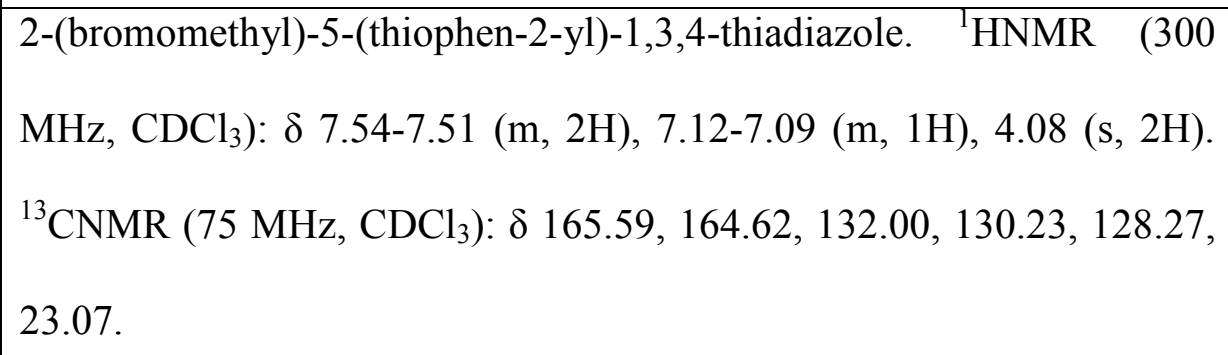 \\
\hline & $\begin{array}{l}\text { methyl 5-(thiophen-2-yl)-1,3,4-oxadiazole-2-carboxylate. } \quad \text { (Yield: } \\
\text { 80\%). }{ }^{1} \mathrm{HNMR}\left(300 \mathrm{MHz}, \mathrm{CDCl}_{3}\right): \delta \text { 7.91-7.89 }(\mathrm{m}, 1 \mathrm{H}), 7.66-7.64(\mathrm{~m},\end{array}$ \\
\hline
\end{tabular}




\begin{tabular}{|c|c|}
\hline & $\begin{array}{l}\text { 1H), 7.26-7.18 (m, 1H), } 4.07(\mathrm{~s}, 3 \mathrm{H}) .{ }^{13} \mathrm{CNMR}\left(75 \mathrm{MHz}, \mathrm{CDCl}_{3}\right): \delta \\
162.85,155.65,154.70,132.17,131.79,128.61,123.82,53.89 .\end{array}$ \\
\hline & $\begin{array}{l}\text { [5-(thiophen-2-yl)-1,3,4-oxadiazol-2-yl]methanol. }{ }^{\mathrm{I}} \mathrm{HNMR}(300 \mathrm{MHz} \text {, } \\
\left.\mathrm{CDCl}_{3}\right): \delta 7.82-7.80(\mathrm{~m}, 1 \mathrm{H}), 7.78-7.76(\mathrm{~m}, 1 \mathrm{H}), 7.24-7.21(\mathrm{~m}, 1 \mathrm{H}) \text {, } \\
4.80(\mathrm{~s}, 2 \mathrm{H}) .\end{array}$ \\
\hline & $\begin{array}{l}\text { methyl 5-(3-methoxythiophen-2-yl)-1,2-oxazole-3-carboxylate. (Yield: } \\
81 \%) .{ }^{1} \mathrm{HNMR}\left(300 \mathrm{MHz}, \mathrm{CDCl}_{3}\right): \delta 7.37(\mathrm{~d}, J=5.49 \mathrm{~Hz}, 1 \mathrm{H}), 6.90 \\
(\mathrm{~s}, 1 \mathrm{H}), 6.88(\mathrm{~d}, J=5.49 \mathrm{~Hz}, 1 \mathrm{H}), 3.98(\mathrm{~s}, 3 \mathrm{H}), 3.96(\mathrm{~s}, 3 \mathrm{H}) .{ }^{13} \mathrm{CNMR} \\
\left(75 \mathrm{MHz}, \mathrm{CDCl}_{3}\right): \delta 166.08,161.23,157.74,157.05,128.05,116.16 \text {, } \\
106.17,100.04,59.36,53.32 .\end{array}$ \\
\hline & $\begin{array}{l}\text { [5-(3-methoxythiophen-2-yl)-1,2-oxazol-3-yl]methanol. (Yield: } 95 \%) \text {. } \\
{ }^{1} \mathrm{HNMR}\left(300 \mathrm{MHz}, \mathrm{CDCl}_{3}\right): \delta 7.33(\mathrm{~d}, J=5.49 \mathrm{~Hz}, 1 \mathrm{H}), 6.89(\mathrm{~d}, J= \\
5.49 \mathrm{~Hz}, 1 \mathrm{H}), 6.59(\mathrm{~s}, 1 \mathrm{H}), 4.78(\mathrm{~d}, J=6.3 \mathrm{~Hz}, 2 \mathrm{H}), 3.98(\mathrm{~s}, 3 \mathrm{H}), 2.46 \\
(\mathrm{t}, J=6.3 \mathrm{~Hz}, 1 \mathrm{H}) .{ }^{13} \mathrm{CNMR}\left(75 \mathrm{MHz}, \mathrm{CDCl}_{3}\right): \delta 164.22,164.18 \text {, } \\
156.74,126.87,115.82,106.73,98.12,58.94,57.34 .\end{array}$ \\
\hline & $\begin{array}{l}\text { methyl 5-[2-methyl-2-(methylsulfanyl)propyl]-1,2-oxazole-3- } \\
\text { carboxylate. (Yield: } 77 \%) .{ }^{1} \mathrm{HNMR}\left(300 \mathrm{MHz}, \mathrm{CDCl}_{3}\right): \delta 6.56(\mathrm{~s}, 1 \mathrm{H}) \text {, } \\
3.96(\mathrm{~s}, 3 \mathrm{H}), 3.03(\mathrm{~s}, 2 \mathrm{H}), 2.06(\mathrm{~s}, 3 \mathrm{H}), 1.32(\mathrm{~s}, 6 \mathrm{H}) .\end{array}$ \\
\hline & $\begin{array}{l}\text { 3-(bromomethyl)-5-[2-methyl-2-(methylsulfanyl)propyl]-1,2-oxazole. } \\
\text { (Yield: } 70 \%) .{ }^{1} \mathrm{HNMR}\left(300 \mathrm{MHz}, \mathrm{CDCl}_{3}\right): \delta 6.24(\mathrm{~s}, 1 \mathrm{H}), 4.41(\mathrm{~s}, \\
2 \mathrm{H}), 2.98(\mathrm{~s}, 2 \mathrm{H}), 2.07(\mathrm{~s}, 3 \mathrm{H}), 1.33(\mathrm{~s}, 6 \mathrm{H}) .\end{array}$ \\
\hline
\end{tabular}

Table S2: Characterizations of final products 


\begin{tabular}{|c|c|}
\hline (6a) & 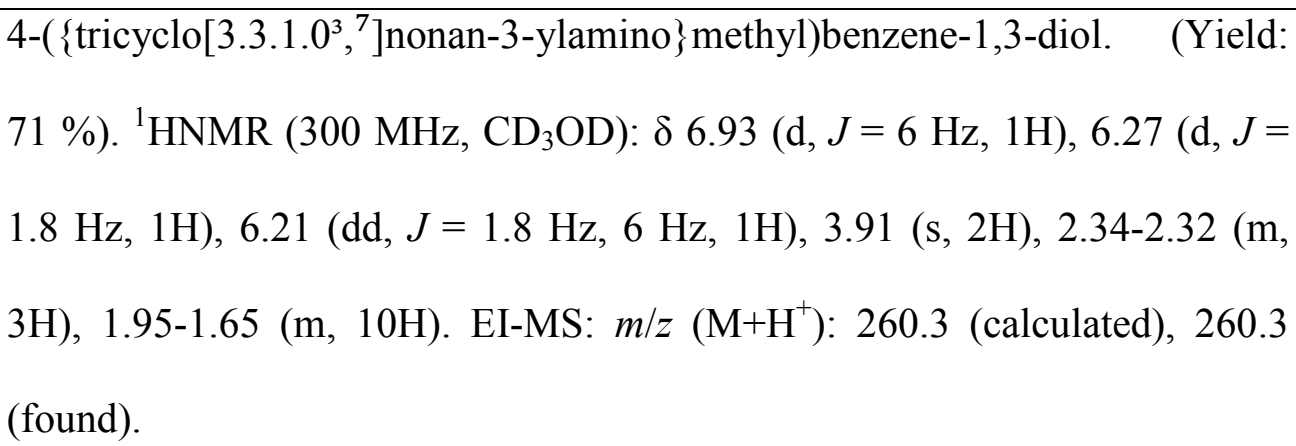 \\
\hline (6b) & $\begin{array}{l}\left.\text { 4- }\left\{4 \text {-azatricyclo[4.3.1.1 }{ }^{3},{ }^{8}\right] \text { undecan-4-ylmethyl }\right\} \text { benzene-1,3-diol. } \quad \text { (Yield: } \\
65 \%) .{ }^{1} \mathrm{HNMR}\left(300 \mathrm{MHz}, \mathrm{CD}_{3} \mathrm{OD}\right): \delta 6.77(\mathrm{~d}, J=6.3 \mathrm{~Hz}, 1 \mathrm{H}), 6.18(\mathrm{~d}, J= \\
1.8 \mathrm{~Hz}, 1 \mathrm{H}), 6.14(\mathrm{dd}, J=1.8 \mathrm{~Hz}, 6 \mathrm{~Hz}, 1 \mathrm{H}), 3.90(\mathrm{~s}, 2 \mathrm{H}), 2.21-2.19(\mathrm{~m}, 1 \mathrm{H}), \\
2.10-1.95(\mathrm{~m}, 6 \mathrm{H}), 1.97-1.90(\mathrm{~m}, 4 \mathrm{H}), 1.76-1.60(\mathrm{~m}, 5 \mathrm{H}) . \text { EI-MS: } m / z \\
\left(\mathrm{M}+\mathrm{H}^{+}\right): 274.4 \text { (calculated), } 274.4 \text { (found). }\end{array}$ \\
\hline (6c) & 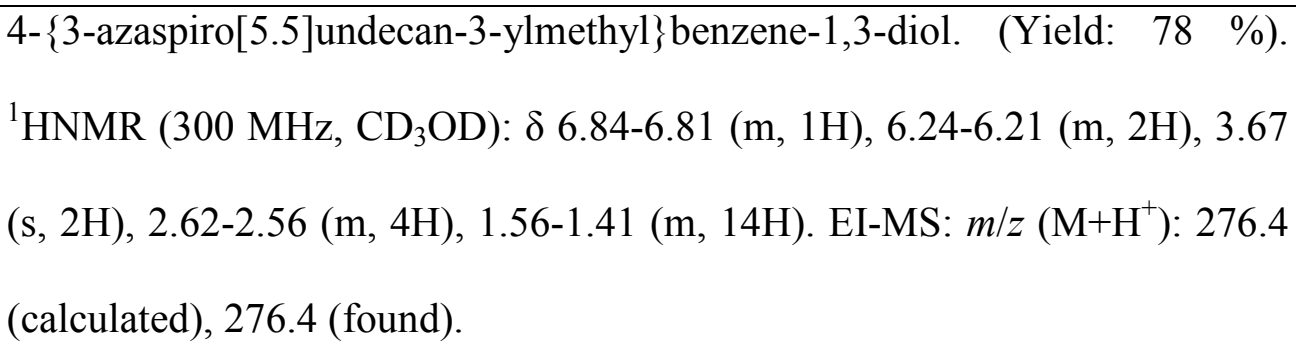 \\
\hline & $\begin{array}{l}\mathrm{N}-(\{5-[4-(\text { methylsulfanyl)phenyl]-1,2-oxazol-3-yl }\} \text { methyl)adamantan-1- } \\
\text { amine. Yield }(85 \%) .{ }^{1} \mathrm{HNMR}\left(300 \mathrm{MHz}, \mathrm{CD}_{3} \mathrm{OD}\right): \delta 7.72(\mathrm{~d}, J=8.52 \mathrm{~Hz} \text {, } \\
2 \mathrm{H}), 7.35(\mathrm{~d}, J=8.52 \mathrm{~Hz}, 2 \mathrm{H}), 6.77(\mathrm{~s}, 1 \mathrm{H}), 3.87(\mathrm{~s}, 2 \mathrm{H}), 2.53(\mathrm{~s}, 3 \mathrm{H}), 2.11- \\
2.08(\mathrm{~m}, 3 \mathrm{H}), 1.77-1.71(\mathrm{~m}, 12 \mathrm{H}) . \mathrm{EI}-\mathrm{MS}: \mathrm{m} / z\left(\mathrm{M}+\mathrm{H}^{+}\right): 355.5 \text { (calculated), } \\
355.3 \text { (found). }\end{array}$ \\
\hline$\Delta \rightarrow$ & $\begin{array}{l}\mathrm{N}-(\{5-[4-(\text { methylsulfanyl)phenyl]-1,2-oxazol-3-yl }\} \text { methyl)tricycle } \\
{\left[3.3 .1 .0^{3},{ }^{7}\right] \text { nonan-3-amine. Yield }(81 \%) .{ }^{1} \mathrm{HNMR}(300 \mathrm{MHz}, \mathrm{DMSO}-d 6): \delta} \\
7.77(\mathrm{~d}, J=7.77 \mathrm{~Hz}, 2 \mathrm{H}), 7.38(\mathrm{~d}, J=7.37 \mathrm{~Hz}, 2 \mathrm{H}), 6.96(\mathrm{~s}, 1 \mathrm{H}), 3.77(\mathrm{~s},\end{array}$ \\
\hline
\end{tabular}




\begin{tabular}{|c|c|}
\hline & $\begin{array}{l}\text { 2H), } 2.53(\mathrm{~s}, 3 \mathrm{H}), 2.22-2.18(\mathrm{~m}, 2 \mathrm{H}), 2.10-2.07(\mathrm{~m}, 1 \mathrm{H}), 1.84-1.68(\mathrm{~m}, 6 \mathrm{H}), \\
1.59-1.46(\mathrm{~m}, 4 \mathrm{H}) . \text { EI-MS: } m / z\left(\mathrm{M}+\mathrm{H}^{+}\right): 325.4 \text { (calculated), } 325.4 \text { (found). }\end{array}$ \\
\hline & 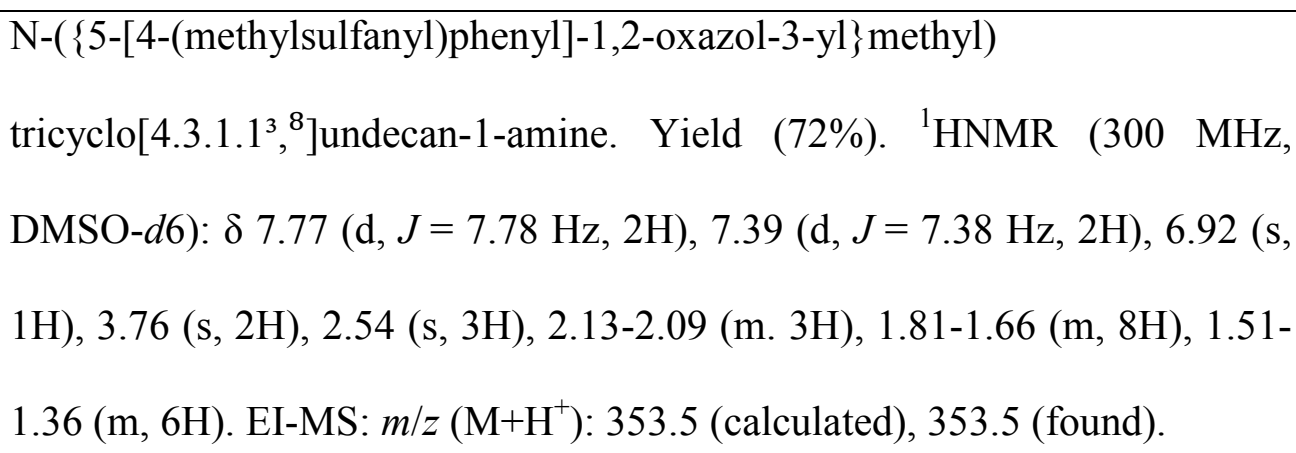 \\
\hline & $\begin{array}{l}\text { (adamantan-1-ylmethyl)( }\{5-[4-(m e t h y l s u l f a n y l) p h e n y l]-1,2-o x a z o l-3- \\
\text { yl }\} \text { methyl)amine. Yield }(73 \%) .{ }^{1} \mathrm{HNMR}\left(300 \mathrm{MHz}, \mathrm{CD}_{3} \mathrm{OD}\right): \delta 7.72(\mathrm{~d}, J= \\
8.52 \mathrm{~Hz}, 2 \mathrm{H}), 7.34(\mathrm{~d}, J=8.52 \mathrm{~Hz}, 2 \mathrm{H}), 6.73(\mathrm{~s}, 1 \mathrm{H}), 3.82(\mathrm{~s}, 2 \mathrm{H}), 2.53(\mathrm{~s}, \\
\text { 3H), } 2.25(\mathrm{~s}, 2 \mathrm{H}), 1.97-1.95(\mathrm{~m}, 3 \mathrm{H}), 1.78-1.66(\mathrm{~m}, 6 \mathrm{H}), 1.57-1.55(\mathrm{~m}, 6 \mathrm{H}) . \\
\text { EI-MS: } m / z\left(\mathrm{M}+\mathrm{H}^{+}\right): 369.5 \text { (calculated), } 369.5 \text { (found). }\end{array}$ \\
\hline ( & $\begin{array}{l}\text { N-[(5-propyl-1,2-oxazol-3-yl)methyl]adamantan-1-amine. Yield (71\%). } \\
{ }^{1} \mathrm{HNMR}\left(300 \mathrm{MHz}, \mathrm{CD}_{3} \mathrm{OD}\right): \delta 6.32(\mathrm{~s}, 1 \mathrm{H}), 4.30(\mathrm{~s}, 2 \mathrm{H}), 2.79(\mathrm{t}, J=7.41 \\
\mathrm{Hz}, 2 \mathrm{H}), 2.25-2.22(\mathrm{~m}, 3 \mathrm{H}), 1.85-1.81(\mathrm{~m}, 6 \mathrm{H}), 1.78-1.71(\mathrm{~m}, 8 \mathrm{H}), 1.00(\mathrm{t}, J \\
=7.35 \mathrm{~Hz}, 3 \mathrm{H}) .{ }^{13} \mathrm{NMR}\left(75 \mathrm{MHz}, \mathrm{CDCl}_{3}\right): \delta 175.48,156.63,100.61,58.06 \text {, } \\
37.87,35.21,34.89,29.34,28.03,20.73,12.55 . \mathrm{EI}-\mathrm{MS}: \mathrm{m} / z\left(\mathrm{M}+\mathrm{H}^{+}\right): 275.4 \\
\text { (calculated), } 275.5 \text { (found). }\end{array}$ \\
\hline 2 & $\begin{array}{l}\text { N-[(5-propyl-1,2,4-oxadiazol-3-yl)methyl]adamantan-1-amine. Yield (81\%). } \\
{ }^{1} \mathrm{HNMR}\left(300 \mathrm{MHz}, \mathrm{CD}_{3} \mathrm{OD}\right): \delta 3.87(\mathrm{~s}, 2 \mathrm{H}), 2.89(\mathrm{t}, J=7.44 \mathrm{~Hz}, 2 \mathrm{H}), 2.10- \\
2.08(\mathrm{~m}, 3 \mathrm{H}), 1.88-1.81(\mathrm{~m}, 2 \mathrm{H}), 1.79-1.65(\mathrm{~m}, 12 \mathrm{H}), 1.02(\mathrm{t}, J=7.38 \mathrm{~Hz} \text {, } \\
\text { 3H). EI-MS: } m / z\left(\mathrm{M}+\mathrm{H}^{+}\right): 276.4 \text { (calculated), } 276.5 \text { (found). }\end{array}$ \\
\hline
\end{tabular}




\begin{tabular}{|c|c|}
\hline (14d) & $\begin{array}{l}\text { N-[(5-cyclobutyl-1,2-oxazol-3-yl)methyl]adamantan-1-amine. } \\
82 \%) .{ }^{1} \mathrm{HNMR}\left(300 \mathrm{MHz}, \mathrm{CD}_{3} \mathrm{OD}\right): \delta 6.02(\mathrm{~s}, 1 \mathrm{H}), 3.81(\mathrm{~s}, 2 \mathrm{H}), 3.62-3.57 \\
(\mathrm{~m}, 1 \mathrm{H}), 2.38-2.32(\mathrm{~m}, 4 \mathrm{H}), 2.09-1.95(\mathrm{~m}, 5 \mathrm{H}), 1.68-1.60(\mathrm{~m}, 12 \mathrm{H}) . \text { EI-MS: } \\
m / z\left(\mathrm{M}+\mathrm{H}^{+}\right): 287.4 \text { (calculated), } 287.4 \text { (found). }\end{array}$ \\
\hline (14e) & $\begin{array}{l}\text { N-\{[5-(methoxymethyl)-1,2-oxazol-3-yl]methyl }\} \text { adamantan-1-amine. } \\
\text { (Yield: } 70 \%) .{ }^{1} \mathrm{HNMR}\left(300 \mathrm{MHz}, \mathrm{CD}_{3} \mathrm{OD}\right): \delta 4.71(\mathrm{~s}, 2 \mathrm{H}), 3.91(\mathrm{~s}, 2 \mathrm{H}) \text {, } \\
3.48(\mathrm{~s}, 3 \mathrm{H}), 2.10-2.07(\mathrm{~m}, 3 \mathrm{H}), 1.74-1.68(\mathrm{~m}, 12 \mathrm{H}) . \mathrm{EI}-\mathrm{MS}: m / z\left(\mathrm{M}+\mathrm{H}^{+}\right): \\
277.4 \text { (calculated), } 277.3 \text { (found). }\end{array}$ \\
\hline (14f) & $\begin{array}{l}\text { N-[(5-isopropyl-1,2-oxazol-3-yl)methyl]adamantan-1-amine. (Yield: } 75 \%) \text {. } \\
{ }^{1} \mathrm{HNMR}\left(300 \mathrm{MHz}, \mathrm{CD}_{3} \mathrm{OD}\right): \delta 3.78(\mathrm{~s}, 2 \mathrm{H}), 3.10-3.03(\mathrm{~m}, 1 \mathrm{H}), 2.09-2.06 \\
(\mathrm{~m}, 3 \mathrm{H}), 1.74-1.70(\mathrm{~m}, 12 \mathrm{H}), 1.32-1.29(\mathrm{~m}, 6 \mathrm{H}) . \mathrm{EI}-\mathrm{MS}: \mathrm{m} / z\left(\mathrm{M}+\mathrm{H}^{+}\right): 275.4 \\
\text { (calculated), } 275.4 \text { (found). }\end{array}$ \\
\hline & $\begin{array}{l}\text { N-\{[5-(2-methylpropyl)-1,2,4-oxadiazol-3-yl]methyl }\} \text { adamantan-1-amine. } \\
\text { (Yield: } 89 \%) .{ }^{1} \mathrm{HNMR}\left(300 \mathrm{MHz}, \mathrm{CD}_{3} \mathrm{OD}\right): \delta 3.87(\mathrm{~s}, 2 \mathrm{H}), 2.80(\mathrm{~d}, J= \\
7.11 \mathrm{~Hz}, 1 \mathrm{H}), 2.24-2.15(\mathrm{~m}, 1 \mathrm{H}), 2.10-2.08(\mathrm{~m}, 3 \mathrm{H}), 1.74-1.72(\mathrm{~m}, 12 \mathrm{H}), \\
1.00(\mathrm{~d}, J=6.69 \mathrm{~Hz}, 6 \mathrm{H}) . \mathrm{EI}-\mathrm{MS}: m / z\left(\mathrm{M}+\mathrm{H}^{+}\right): 290.4 \text { (calculated), } 290.6 \\
\text { (found). }\end{array}$ \\
\hline & $\begin{array}{l}\mathrm{N}-(\{5-[2-m e t h y l-2-(m e t h y l s u l f a n y l) p r o p y l]-1,2-o x a z o l-3-y l\} \text { methyl) } \\
\text { adamantan-1-amine. (Yield: } 88 \%) .{ }^{1} \mathrm{HNMR}(300 \mathrm{MHz}, \mathrm{DMSO}-d 6): \delta 6.31 \\
(\mathrm{~s}, 1 \mathrm{H}), 3.70(\mathrm{~s}, 2 \mathrm{H}), 2.98(\mathrm{~s}, 2 \mathrm{H}), 2.04(\mathrm{~s}, 3 \mathrm{H}), 2.03-2.01(\mathrm{~m}, 3 \mathrm{H}), 1.65-1.55 \\
(\mathrm{~m}, 12 \mathrm{H}), 1.27(\mathrm{~s}, 6 \mathrm{H}) . \mathrm{EI}-\mathrm{MS}: \mathrm{m} / z\left(\mathrm{M}+\mathrm{H}^{+}\right): 335.5 \text { (calculated), } 335.5 \\
\text { (found). }\end{array}$ \\
\hline
\end{tabular}




\begin{tabular}{|c|c|}
\hline (14h) & $\begin{array}{l}\text { N-[(5-cyclohexyl-1,2-oxazol-3-yl)methyl]adamantan-1-amine. (Yield: } \\
75 \%) .{ }^{1} \mathrm{HNMR}\left(300 \mathrm{MHz}, \mathrm{CD}_{3} \mathrm{OD}\right): \delta 6.27(\mathrm{~s}, 1 \mathrm{H}), 4.29(\mathrm{~s}, 2 \mathrm{H}), 3.32-3.30 \\
(\mathrm{~m}, 1 \mathrm{H}), 2.90-2.81(\mathrm{~m}, 3 \mathrm{H}), 2.24-2.20(\mathrm{~m}, 8 \mathrm{H}), 1.85-1.73(\mathrm{~m}, 9 \mathrm{H}), 1.37-1.29 \\
(\mathrm{~m}, 5 \mathrm{H}) . \text { EI-MS: } m / z\left(\mathrm{M}+\mathrm{H}^{+}\right): 315.5 \text { (calculated), } 315.7 \text { (found). }\end{array}$ \\
\hline (14i) & $\begin{array}{l}\text { N-\{[5-(thiophen-3-yl)-1,2-oxazol-3-yl]methyl }\} \text { adamantan-1-amine. (Yield: } \\
82 \%) .{ }^{1} \mathrm{HNMR}(300 \mathrm{MHz}, \mathrm{DMSO}-d 6): \delta 8.12-8.11(\mathrm{~m}, 1 \mathrm{H}), 7.74-7.71(\mathrm{~m} \text {, } \\
1 \mathrm{H}), 7.56-7.54(\mathrm{~m}, 1 \mathrm{H}), 6.8(\mathrm{~s}, 1 \mathrm{H}), 3.73(\mathrm{~s}, 2 \mathrm{H}), 2.10-2.07(\mathrm{~m}, 3 \mathrm{H}), 1.63- \\
1.60(\mathrm{~m}, 12 \mathrm{H}) . \text { EI-MS: } \mathrm{m} / z\left(\mathrm{M}+\mathrm{H}^{+}\right): 315.4 \text { (calculated), } 315.1 \text { (found). }\end{array}$ \\
\hline (18g) & $\begin{array}{l}\text { N- }\{[5-(\text { thiophen-3-yl)-1,2,4-oxadiazol-3-yl }] \text { methyl }\} \text { adamantan-1-amine. } \\
\text { (Yield: } 77 \%) .{ }^{1} \mathrm{HNMR}\left(300 \mathrm{MHz}, \mathrm{CD}_{3} \mathrm{OD}\right): \delta 8.40-89.39(\mathrm{~m}, 1 \mathrm{H}), 7.70- \\
7.65(\mathrm{~m}, 2 \mathrm{H}), 3.93(\mathrm{~s}, 2 \mathrm{H}), 2.10-2.08(\mathrm{~m}, 3 \mathrm{H}), 1.76-1.70(\mathrm{~m}, 12 \mathrm{H}) .{ }^{13} \mathrm{CNMR} \\
(75 \mathrm{MHz}, \mathrm{CD} 3 \mathrm{OD}): 173.45,171.34,131.96,129.31,127.35,126.72,52.35 \text {, } \\
42.78,37.58,37.04,30.98 . \mathrm{EI}-\mathrm{MS}: m / z\left(\mathrm{M}+\mathrm{H}^{+}\right): 316.4 \text { (calculated), } 316.7 \\
\text { (found). }\end{array}$ \\
\hline (14j) & $\begin{array}{l}\text { N-\{[5-(furan-2-yl)-1,2-oxazol-3-yl]methyl }\} \text { adamantan-1-amine. (Yield: } \\
77 \%) .{ }^{1} \mathrm{HNMR}(300 \mathrm{MHz}, \mathrm{DMSO}-d 6): \delta 7.96(\mathrm{~s}, 1 \mathrm{H}), 7.14-7.12(\mathrm{~m}, 1 \mathrm{H}) \text {, } \\
6.78-6.75(\mathrm{~m}, 2 \mathrm{H}), 3.79(\mathrm{~m}, 2 \mathrm{H}), 2.06-2.04(\mathrm{~m}, 3 \mathrm{H}), 1.63-1.60(\mathrm{~m}, 12 \mathrm{H}) . \text { EI- } \\
\text { MS: } m / z\left(\mathrm{M}+\mathrm{H}^{+}\right): 299.4 \text { (calculated), } 299.2 \text { (found). }\end{array}$ \\
\hline$E$ & 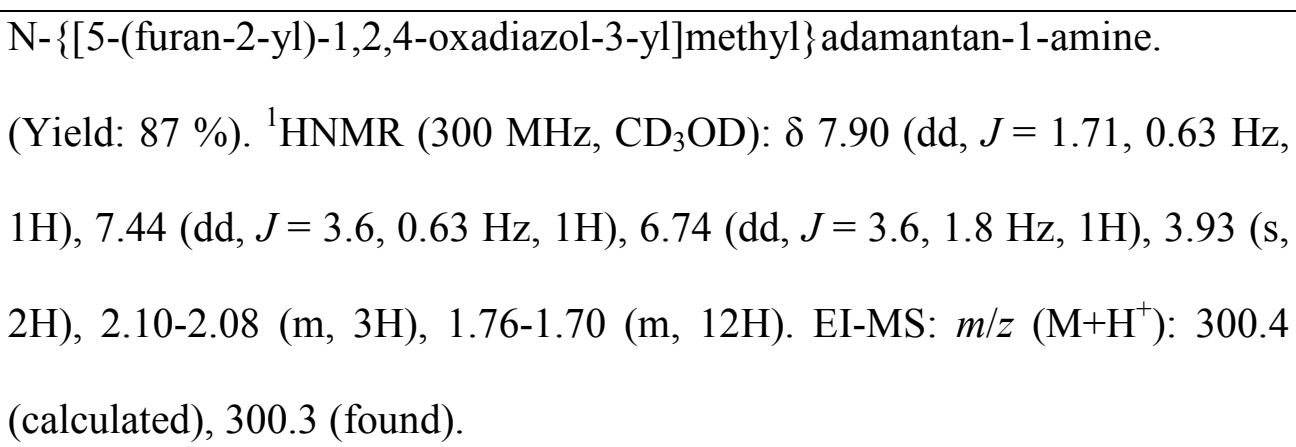 \\
\hline
\end{tabular}




\begin{tabular}{|c|c|}
\hline ( & $\begin{array}{l}\mathrm{N}-\{[5-(3-m e t h o x y t h i o p h e n-2-y l)-1,2-\text { oxazol-3-yl }] \text { methyl }\} \text { adamantan-1- } \\
\text { amine. (Yield: } 82 \%) .{ }^{1} \mathrm{HNMR}\left(300 \mathrm{MHz}, \mathrm{CD}_{3} \mathrm{OD}\right): \delta 7.55(\mathrm{~d}, J=5.46 \mathrm{~Hz} \text {, } \\
1 \mathrm{H}), 7.10(\mathrm{~d}, J=5.46 \mathrm{~Hz}, 1 \mathrm{H}), 6.70(\mathrm{~s}, 1 \mathrm{H}), 4.03(\mathrm{~s}, 3 \mathrm{H}), 3.85(\mathrm{~s}, 2 \mathrm{H}), 2.12- \\
2.09(\mathrm{~m}, 2 \mathrm{H}), 1.77-1.72(\mathrm{~m}, 12 \mathrm{H}) . \mathrm{EI}-\mathrm{MS}: m / z\left(\mathrm{M}+\mathrm{H}^{+}\right): 345.5 \text { (calculated), } \\
345.5 \text { (found). }\end{array}$ \\
\hline & $\begin{array}{l}\text { N-\{[5-(2-chlorophenyl)-1,2-oxazol-3-yl]methyl }\} \text { adamantan-1-amine. } \\
\text { (Yield: } 75 \%) .{ }^{1} \mathrm{HNMR}(300 \mathrm{MHz}, \mathrm{DMSO}-d 6): \delta 7.94-7.86(\mathrm{~m}, 1 \mathrm{H}), 7.70- \\
7.63(\mathrm{~m}, 1 \mathrm{H}), 7.59-7.47(\mathrm{~m}, 2 \mathrm{H}), 7.06(\mathrm{~s}, 1 \mathrm{H}), 3.80(\mathrm{~s}, 2 \mathrm{H}), 2.04-2.02(\mathrm{~m}, \\
3 \mathrm{H}), 1.65-1.56(\mathrm{~m}, 12 \mathrm{H}) . \mathrm{EI}-\mathrm{MS}: m / z\left(\mathrm{M}+\mathrm{H}^{+}\right): 343.9 \text { (calculated), } 343.8 \\
\text { (found). }\end{array}$ \\
\hline ( & $\begin{array}{l}\text { N- }\{[5-(2-c h l o r o p h e n y l)-1,2,4-o x a d i a z o l-3-y l] m e t h y l\} \text { adamantan-1-amine. } \\
\text { (Yield: } 84 \%) .{ }^{1} \mathrm{HNMR}(300 \mathrm{MHz}, \mathrm{DMSO}-d 6): \delta 8.10-8.04(\mathrm{~m}, 1 \mathrm{H}), 7.78- \\
7.65(\mathrm{~m}, 2 \mathrm{H}), 7.59(\mathrm{ddd}, J=7.9,7.1,1.5 \mathrm{~Hz}, 1 \mathrm{H}), 3.91(\mathrm{~s}, 2 \mathrm{H}), 2.06-2.02 \\
(\mathrm{~m}, 3 \mathrm{H}), 1.88(\mathrm{br} \mathrm{s}, 1 \mathrm{H}), 1.64-1.56(\mathrm{~m}, 12 \mathrm{H}) . \mathrm{EI}-\mathrm{MS}: \mathrm{m} / z\left(\mathrm{M}+\mathrm{H}^{+}\right): 344.9 \\
\text { (calculated), } 344.8 \text { (found). }\end{array}$ \\
\hline & $\begin{array}{l}\text { N- }\{[5-(2-\text { bromophenyl)-1,2-oxazol-3-yl }] \text { methyl }\} \text { adamantan-1-amine. } \\
\text { (Yield: } 84 \%) .{ }^{1} \mathrm{HNMR}(300 \mathrm{MHz}, \mathrm{CD} 3 \mathrm{OD}): \delta 7.84-7.78(\mathrm{~m}, 2 \mathrm{H}), 7.54-7.52 \\
(\mathrm{~m}, 1 \mathrm{H}), 7.49-7.42(\mathrm{~m}, 1 \mathrm{H}), 7.12(\mathrm{~s}, 1 \mathrm{H}), 3.92(\mathrm{~s}, 2 \mathrm{H}), 2.12-2.09(\mathrm{~m}, 3 \mathrm{H}), \\
1.79-1.73(\mathrm{~m}, 12 \mathrm{H}) . \text { EI-MS: } \mathrm{m} / z\left(\mathrm{M}+\mathrm{H}^{+}\right): 388.1 \text { (calculated), } 388.1 \text { (found). }\end{array}$ \\
\hline (140) & $\begin{array}{l}\text { N- }\{[5-(3,5-d i f l u o r o p h e n y l)-1,2-o x a z o l-3-y l] m e t h y l\} \text { adamantan-1-amine. } \\
\text { (Yield: } 89 \%) .{ }^{1} \mathrm{HNMR}(300 \mathrm{MHz}, \mathrm{CD} 3 \mathrm{OD}): \delta 7.49-7.42(\mathrm{~m}, 2 \mathrm{H}), 7.14-7.08 \\
(\mathrm{~m}, 1 \mathrm{H}), 6.94(\mathrm{~s}, 1 \mathrm{H}), 3.89(\mathrm{~s}, 2 \mathrm{H}), 2.11-2.08(\mathrm{~m}, 3 \mathrm{H}), 1.77-1.67(\mathrm{~m}, 12 \mathrm{H}) . \\
\text { EI-MS: } m / z\left(\mathrm{M}+\mathrm{H}^{+}\right): 345.4 \text { (calculated), } 345.4 \text { (found). }\end{array}$ \\
\hline
\end{tabular}




\begin{tabular}{|c|c|}
\hline & $\begin{array}{l}\text { N-\{[5-(2,6-difluorophenyl)-1,2-oxazol-3-yl]methyl }\} \text { adamantan-1-amine. } \\
\text { (Yield: } 79 \%) .{ }^{1} \mathrm{HNMR}\left(300 \mathrm{MHz}, \mathrm{CD}_{3} \mathrm{OD}\right): \delta 7.58-7.52(\mathrm{~m}, 1 \mathrm{H}), 7.20-7.14 \\
(\mathrm{~m}, 2 \mathrm{H}), 6.91-6.90(\mathrm{~m}, 1 \mathrm{H}), 3.92(\mathrm{~s}, 2 \mathrm{H}), 2.10-2.07(\mathrm{~m}, 3 \mathrm{H}), 1.78-1.71(\mathrm{~m}, \\
\text { 12H). EI-MS: } m / z\left(\mathrm{M}+\mathrm{H}^{+}\right): 345.4 \text { (calculated), } 345.4 \text { (found). }\end{array}$ \\
\hline & $\begin{array}{l}\text { N-\{[5-(3-methoxyphenyl)-1,2-oxazol-3-yl]methyl }\} \text { adamantan-1-amine. } \\
\text { (Yield: } 81 \%) .{ }^{1} \mathrm{HNMR}\left(300 \mathrm{MHz}, \mathrm{CD}_{3} \mathrm{OD}\right): \delta 7.41-7.34(\mathrm{~m}, 3 \mathrm{H}), 7.06-7.01 \\
(\mathrm{~m}, 1 \mathrm{H}), 6.82(\mathrm{~s}, 1 \mathrm{H}), 3.87(\mathrm{~s}, 2 \mathrm{H}), 3.86(\mathrm{~s}, 3 \mathrm{H}), 2.10-2.08(\mathrm{~m}, 3 \mathrm{H}), 1.77- \\
1.71(\mathrm{~m}, 12 \mathrm{H}) . \text { EI-MS: } m / z\left(\mathrm{M}+\mathrm{H}^{+}\right): 339.5 \text { (calculated), } 339.5 \text { (found). }\end{array}$ \\
\hline$(1$ & 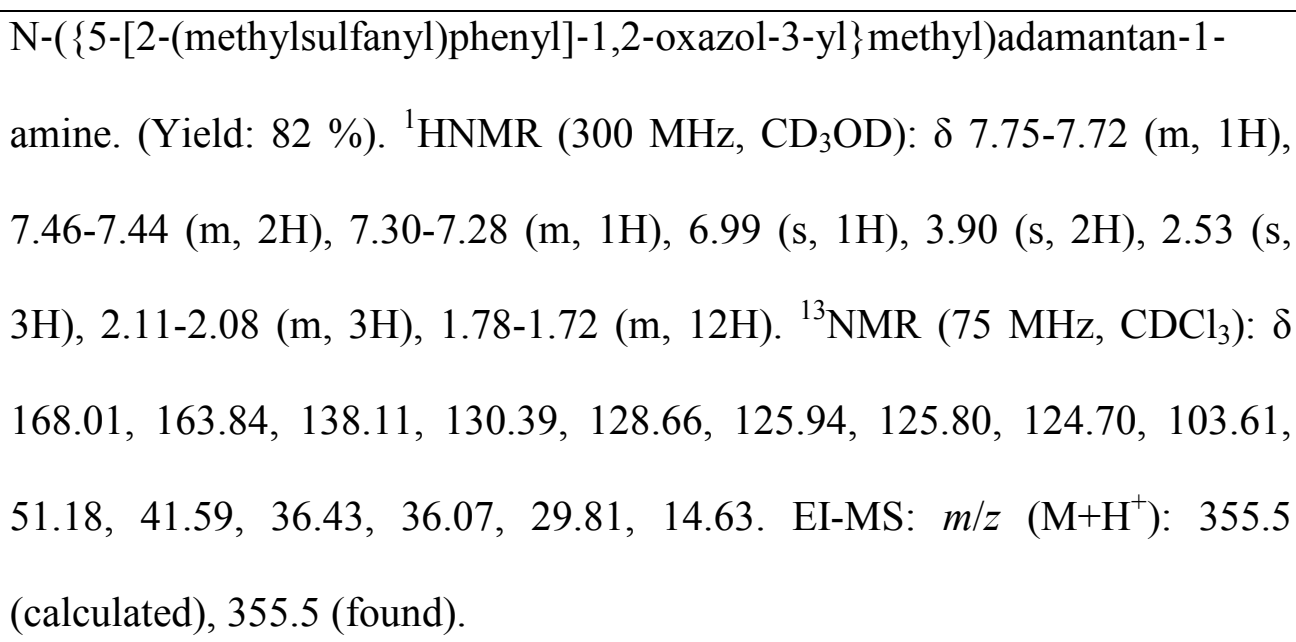 \\
\hline (181) & $\begin{array}{l}\text { N- }\{[5-(2-m e t h o x y p h e n y l)-1,2,4-o x a d i a z o l-3-y l] m e t h y l\} \text { adamantan-1-amine. } \\
\text { (Yield: } 85 \%) .{ }^{1} \mathrm{HNMR}(300 \mathrm{MHz}, \mathrm{CD} 3 \mathrm{OD}): \delta 8.04-8.01(\mathrm{~m}, 1 \mathrm{H}), 7.66-7.60 \\
(\mathrm{~m}, 1 \mathrm{H}), 7.26-7.23(\mathrm{~m}, 1 \mathrm{H}), 7.15-7.10(\mathrm{~m}, 1 \mathrm{H}), 3.97(\mathrm{~s}, 3 \mathrm{H}), 3.93(\mathrm{~s}, 2 \mathrm{H}), \\
2.11-2.09(\mathrm{~m}, 3 \mathrm{H}), 1.77-1.72(\mathrm{~m}, 12 \mathrm{H}) . \mathrm{EI}-\mathrm{MS}: \mathrm{m} / z\left(\mathrm{M}+\mathrm{H}^{+}\right): 340.4 \\
\text { (calculated), } 340.4 \text { (found). }\end{array}$ \\
\hline$(14 t)$ & $\begin{array}{l}\text { N- }\{[5-(2,4-d i m e t h o x y p h e n y l)-1,2-o x a z o l-3-y l] m e t h y l\} \text { adamantan-1-amine. } \\
\text { (Yield: } 91 \%) .{ }^{1} \mathrm{HNMR}(300 \mathrm{MHz}, \mathrm{DMSO}-d 6): \delta 7.75(\mathrm{~d}, J=8.6 \mathrm{~Hz}, 1 \mathrm{H}) \text {, } \\
6.78-6.62(\mathrm{~m}, 3 \mathrm{H}), 3.95(\mathrm{~s}, 3 \mathrm{H}), 3.85(\mathrm{~s}, 3 \mathrm{H}), 3.75(\mathrm{~s}, 2 \mathrm{H}), 2.05-2.02(\mathrm{~m} \text {, }\end{array}$ \\
\hline
\end{tabular}




\begin{tabular}{|c|c|}
\hline & $\begin{array}{l}3 \mathrm{H}), 1.66-1.57(\mathrm{~m}, 12 \mathrm{H}) . \text { EI-MS: } \mathrm{m} / z\left(\mathrm{M}+\mathrm{H}^{+}\right): 369.5 \text { (calculated), } 369.4 \\
\text { (found). }\end{array}$ \\
\hline$(18 \mathrm{~m})$ & $\begin{array}{l}\mathrm{N}-\{[5-(2,4-d i m e t h o x y p h e n y l)-1,2,4 \text {-oxadiazol-3-yl]methyl }\} \text { adamantan-1- } \\
\text { amine. (Yield: } 62 \%) .{ }^{1} \mathrm{HNMR}\left(300 \mathrm{MHz}, \mathrm{CD}_{3} \mathrm{OD}\right): \delta 8.08-8.05(\mathrm{~m}, 1 \mathrm{H}) \text {, } \\
6.76-6.72(\mathrm{~m}, 2 \mathrm{H}), 4.45(\mathrm{~s}, 2 \mathrm{H}(, 3.99(\mathrm{~s}, 3 \mathrm{H}), 3.92(\mathrm{~s}, 3 \mathrm{H}), 2.27-2.25(\mathrm{~m}, \\
3 \mathrm{H}), 2.04-2.02(\mathrm{~m}, 6 \mathrm{H}), 1.82-1.75(\mathrm{~m}, 6 \mathrm{H}) . \mathrm{EI-MS}: \mathrm{m} / \mathrm{z}\left(\mathrm{M}+\mathrm{H}^{+}\right): 370.5 \\
\text { (calculated), } 370.5 \text { (found). }\end{array}$ \\
\hline$(202$ & $\begin{array}{l}\mathrm{N}-\{[3-(3,4-d i m e t h o x y p h e n y l)-1,2,4-\text { oxadiazol-5-yl]methyl }\} \text { adamantan-1- } \\
\text { amine. (Yield: } 72 \%) .{ }^{1} \mathrm{HNMR}\left(300 \mathrm{MHz}, \mathrm{CD}_{3} \mathrm{OD}\right): \delta 7.70-7.67(\mathrm{~m}, 1 \mathrm{H}) \text {, } \\
7.64-7.61(\mathrm{~m}, 1 \mathrm{H}), 7.10-7.07(\mathrm{~m}, 1 \mathrm{H}), 4.10(\mathrm{~s}, 2 \mathrm{H}), 3.91(\mathrm{~s}, 6 \mathrm{H}), 2.12-2.09 \\
(\mathrm{~m}, 3 \mathrm{H}), 1.77-1.74(\mathrm{~m}, 12 \mathrm{H}) . \mathrm{EI}-\mathrm{MS}: \mathrm{m} / z\left(\mathrm{M}+\mathrm{H}^{+}\right): 370.5 \text { (calculated), } 370.5 \\
\text { (found). }\end{array}$ \\
\hline & $\begin{array}{l}\text { N-\{[3-(2-methoxyphenyl)-1,2,4-oxadiazol-5-yl]methyl }\} \text { adamantan-1-amine. } \\
\text { (Yield: } 79 \%) .{ }^{1} \mathrm{HNMR}(300 \mathrm{MHz}, \mathrm{DMSO}-\mathrm{d} 6): \delta 7.82(\mathrm{dd}, \mathrm{J}=7.6,1.7 \mathrm{~Hz}, \\
1 \mathrm{H}), 7.55(\mathrm{ddd}, \mathrm{J}=8.4,7.3,1.8 \mathrm{~Hz}, 1 \mathrm{H}), 7.22(\mathrm{dd}, \mathrm{J}=8.4,1.0 \mathrm{~Hz}, 1 \mathrm{H}), 7.11 \\
(\mathrm{td}, \mathrm{J}=7.5,1.1 \mathrm{~Hz}, 1 \mathrm{H}), 4.05(\mathrm{~s}, 2 \mathrm{H}), 3.87(\mathrm{~s}, 3 \mathrm{H}), 2.22(\mathrm{br} \mathrm{s}, 1 \mathrm{H}), 2.11- \\
1.94(\mathrm{~m}, 3 \mathrm{H}), 1.70-1.45(\mathrm{~m}, 12 \mathrm{H}) . \mathrm{EI}-\mathrm{MS}: \mathrm{m} / z\left(\mathrm{M}+\mathrm{H}^{+}\right): 340.4 \text { (calculated), } \\
340.4 \text { (found). }\end{array}$ \\
\hline 4 & $\begin{array}{l}\mathrm{N}-[(3-\text { cyclohexyl-1,2-oxazol-5-yl)methyl]adamantan-1-amine. } \\
77 \%) .{ }^{1} \mathrm{HNMR}(300 \mathrm{MHz}, \mathrm{DMSO}-d 6): \delta 6.22(\mathrm{~s}, 1 \mathrm{H}), 3.76(\mathrm{~s}, 2 \mathrm{H}), 2.69- \\
2.62(\mathrm{~m}, 1 \mathrm{H}), 2.04-2.01(\mathrm{~m}, 3 \mathrm{H}), 1.88-1.83(\mathrm{~m}, 3 \mathrm{H}), 1.77-1.72(\mathrm{~m}, 2 \mathrm{H}), \\
1.65-1.55(\mathrm{~m}, 12 \mathrm{H}), 1.46-1.31(\mathrm{~m}, 5 \mathrm{H}) . \text { EI-MS: } m / z\left(\mathrm{M}+\mathrm{H}^{+}\right): 315.5 \\
\text { (calculated), } 315.5 \text { (found). }\end{array}$ \\
\hline
\end{tabular}




\begin{tabular}{|c|c|}
\hline (26a) & $\begin{array}{l}\text { N-\{[5-(thiophen-2-yl)-1,3,4-oxadiazol-2-yl]methyl }\} \text { adamantan-1-amine. } \\
\text { (Yield: } 72 \%) .{ }^{1} \mathrm{HNMR}\left(300 \mathrm{MHz}, \mathrm{CD}_{3} \mathrm{OD}\right): \delta 7.96-7.92(\mathrm{~m}, 1 \mathrm{H}), 7.82-7.80 \\
(\mathrm{~m}, 1 \mathrm{H}), 7.31-7.26(\mathrm{~m}, 1 \mathrm{H}), 3.96(\mathrm{~s}, 2 \mathrm{H}), 2.02-1.99(\mathrm{~m}, 3 \mathrm{H}), 1.59-1.56(\mathrm{~m}, \\
\text { 12H). EI-MS: } m / z\left(\mathrm{M}+\mathrm{H}^{+}\right): 316.4 \text { (calculated), } 316.5 \text { (found). }\end{array}$ \\
\hline (2) & $\begin{array}{l}\text { N-[(5-phenyl-1,3,4-oxadiazol-2-yl)methyl]adamantan-1-amine. } \\
88 \%) .{ }^{1} \mathrm{HNMR}(300 \mathrm{MHz}, \mathrm{DMSO}-d 6): \delta 8.04-8.01(\mathrm{~m}, 2 \mathrm{H}), 7.66-7.63(\mathrm{~m}, \\
3 \mathrm{H}), 4.03(\mathrm{~s}, 2 \mathrm{H}), 2.25(\text { br s, } 1 \mathrm{H}), 2.07-2.04(\mathrm{~m}, 3 \mathrm{H}), 1.65-1.62(\mathrm{~m}, 12 \mathrm{H}) . \\
\text { EI-MS: } m / z\left(\mathrm{M}+\mathrm{H}^{+}\right): 310.4 \text { (calculated), } 310.5 \text { (found). }\end{array}$ \\
\hline$(26$ & $\begin{array}{l}\mathrm{N}-\{[5-(4-m e t h o x y p h e n y l)-1,3,4-o x a d i a z o l-2-y l] m e t h y l\} \text { adamantan-1-amine. } \\
\text { (Yield: } 82 \%) .{ }^{1} \mathrm{HNMR}(300 \mathrm{MHz}, \mathrm{DMSO}-d 6): \delta 7.92(\mathrm{~d}, J=9 \mathrm{~Hz}, 2 \mathrm{H}) \text {, } \\
7.13(\mathrm{~d}, J=9 \mathrm{~Hz}, 2 \mathrm{H}), 3.96(\mathrm{~s}, 2 \mathrm{H}), 3.84(\mathrm{~s}, 3 \mathrm{H}), 2.04-2.00(\mathrm{~m}, 3 \mathrm{H}), 1.63- \\
1.54(\mathrm{~m}, 12 \mathrm{H}) . \text { EI-MS: } m / z\left(\mathrm{M}+\mathrm{H}^{+}\right): 340.4 \text { (calculated), } 340.4 \text { (found). }\end{array}$ \\
\hline (26b) & $\begin{array}{l}\text { N- }\{[5 \text {-(thiophen-2-yl)-1,3,4-thiadiazol-2-yl]methyl }\} \text { adamantan-1-amine. } \\
\text { (Yield: } 80 \%) .{ }^{1} \mathrm{HNMR}(300 \mathrm{MHz}, \mathrm{DMSO}-d 6): \delta 7.78-7.71(\mathrm{~m}, 2 \mathrm{H}), 7.20- \\
7.17(\mathrm{~m}, 1 \mathrm{H}), 4.06(\mathrm{~s}, 2 \mathrm{H}), 2.02-1.99(\mathrm{~m}, 3 \mathrm{H}), 1.62-1.56(\mathrm{~m}, 12 \mathrm{H}) . \text { EI-MS: } \\
m / z\left(\mathrm{M}+\mathrm{H}^{+}\right): 332.5 \text { (calculated), } 332.2 \text { (found). }\end{array}$ \\
\hline (26e) & $\begin{array}{l}\text { N- }\{[2-(\text { thiophen-2-yl)-1,3-thiazol-5-yl]methyl }\} \text { adamantan-1-amine. (Yield: } \\
\text { 89\%). }{ }^{1} \mathrm{HNMR}(300 \mathrm{MHz}, \mathrm{DMSO}-d 6): \delta 7.69-7.60(\mathrm{~m}, 2 \mathrm{H}), 7.58-7.54(\mathrm{~m}, \\
\text { 1H), 7.15-7.13 (m, } 1 \mathrm{H}), 3.92(\mathrm{~s}, 2 \mathrm{H}), 2.06-2.02(\mathrm{~m}, 3 \mathrm{H}), 1.65-1.56(\mathrm{~m}, \\
\text { 12H). EI-MS: } m / z\left(\mathrm{M}+\mathrm{H}^{+}\right): 331.5 \text { (calculated), } 331.5 \text { (found). }\end{array}$ \\
\hline & $\begin{array}{l}\mathrm{N}-\{[2-(\text { thiophen-2-yl)-1,3-thiazol-4-yl]methyl }\} \text { adamantan-1-amine. (Yield: } \\
70 \%) .{ }^{1} \mathrm{HNMR}\left(300 \mathrm{MHz}, \mathrm{CD}_{3} \mathrm{OD}\right): \delta 7.60-7.55(\mathrm{~m}, 2 \mathrm{H}), 7.34(\mathrm{~s}, 1 \mathrm{H}) \text {, } \\
7.14-7.11(\mathrm{~m}, 1 \mathrm{H}), 3.92(\mathrm{~s}, 2 \mathrm{H}), 2.12-2.09(\mathrm{~m}, 3 \mathrm{H}), 1.81-1.72(\mathrm{~m}, 12 \mathrm{H}) . \text { EI- }\end{array}$ \\
\hline
\end{tabular}




\begin{tabular}{|c|c|}
\hline & MS: $m / z\left(\mathrm{M}+\mathrm{H}^{+}\right): 331.5$ (calculated), 331.5 (found). \\
\hline (26i) & $\begin{array}{l}\text { N-[(2-phenyl-1,3-thiazol-4-yl)methyl]adamantan-1-amine. (Yield: } 62 \%) \text {. } \\
{ }^{1} \mathrm{HNMR}\left(300 \mathrm{MHz}, \mathrm{CD}_{3} \mathrm{OD}\right): \delta 8.02-7.98(\mathrm{~m}, 2 \mathrm{H}), 7.63(\mathrm{~s}, 1 \mathrm{H}), 7.51-7.48 \\
(\mathrm{~m}, 3 \mathrm{H}), 4.27(\mathrm{~s}, 2 \mathrm{H}), 2.23-2.19(\mathrm{~m}, 3 \mathrm{H}), 2.01-1.97(\mathrm{~m}, 6 \mathrm{H}), 1.80-1.77(\mathrm{~m}, \\
6 \mathrm{H}) . \text { EI-MS: } m / z\left(\mathrm{M}+\mathrm{H}^{+}\right): 325.5 \text { (calculated), } 325.3 \text { (found). }\end{array}$ \\
\hline$(30$ & 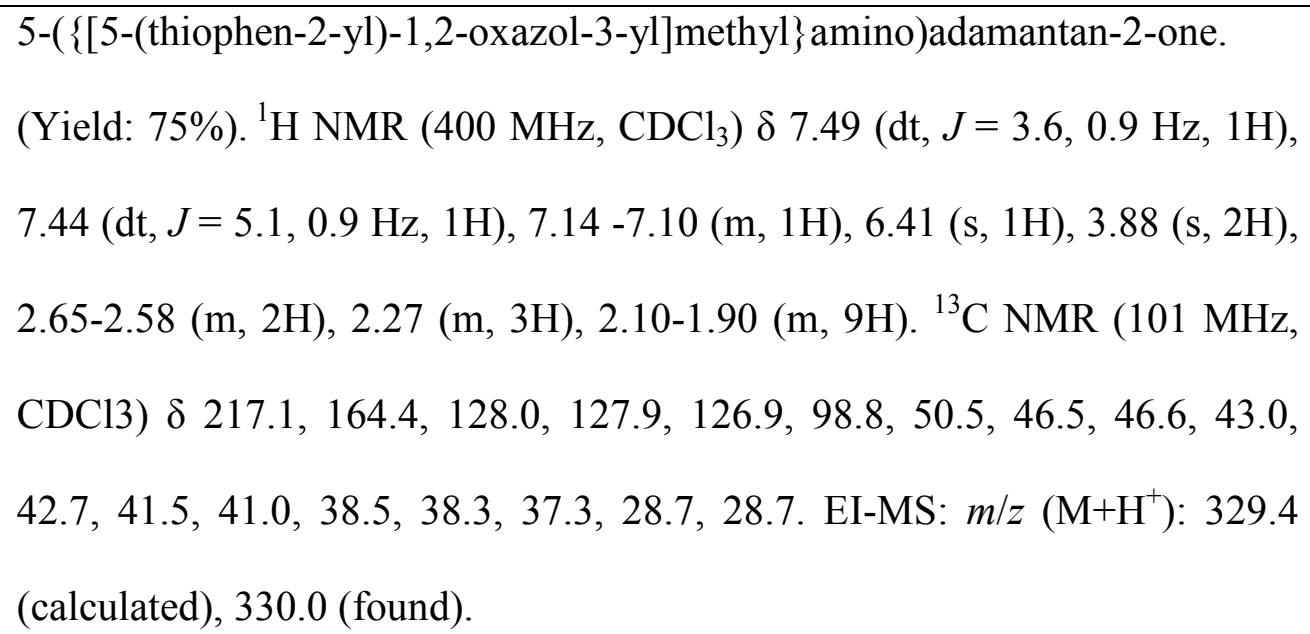 \\
\hline & 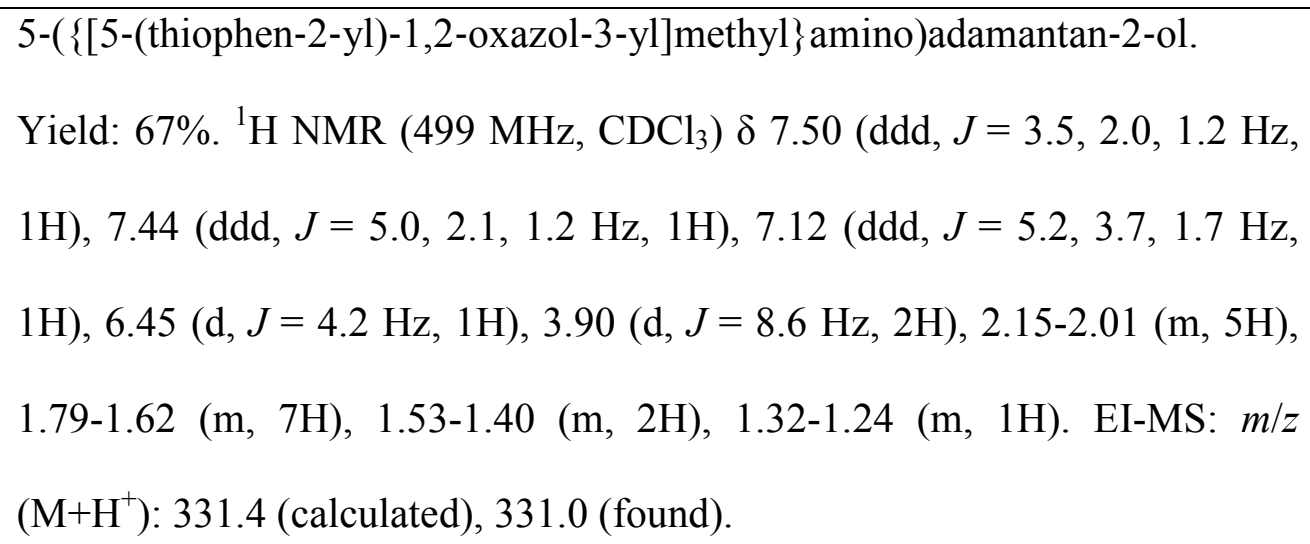 \\
\hline & 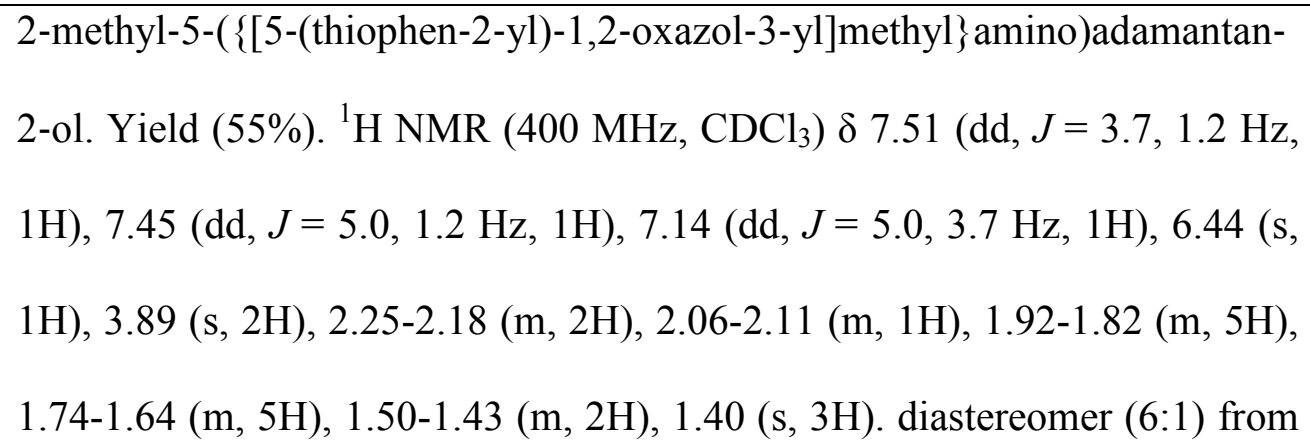 \\
\hline
\end{tabular}




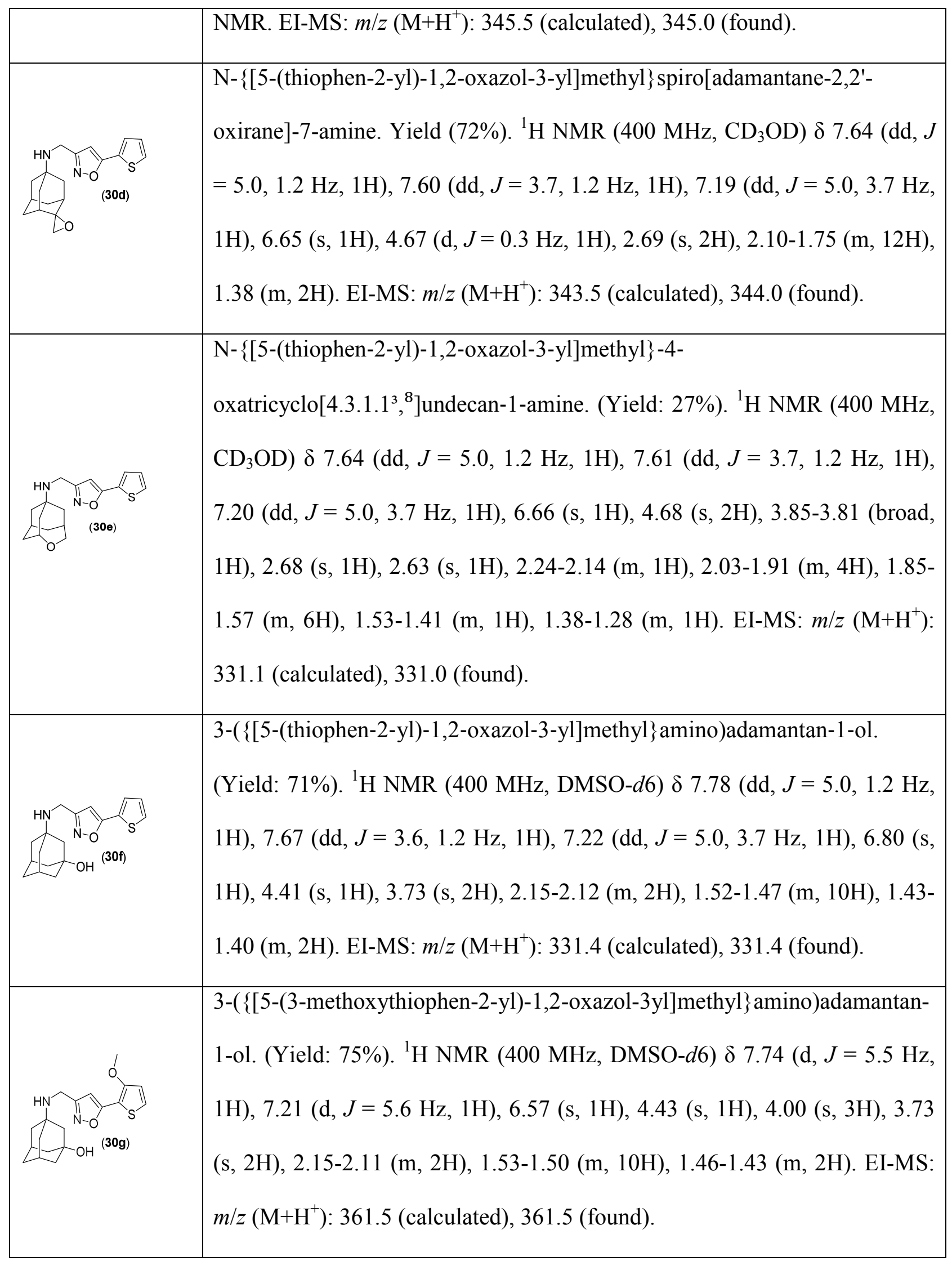




\begin{tabular}{|c|c|}
\hline $\mathrm{COH}^{(30 \mathrm{~h})}$ & 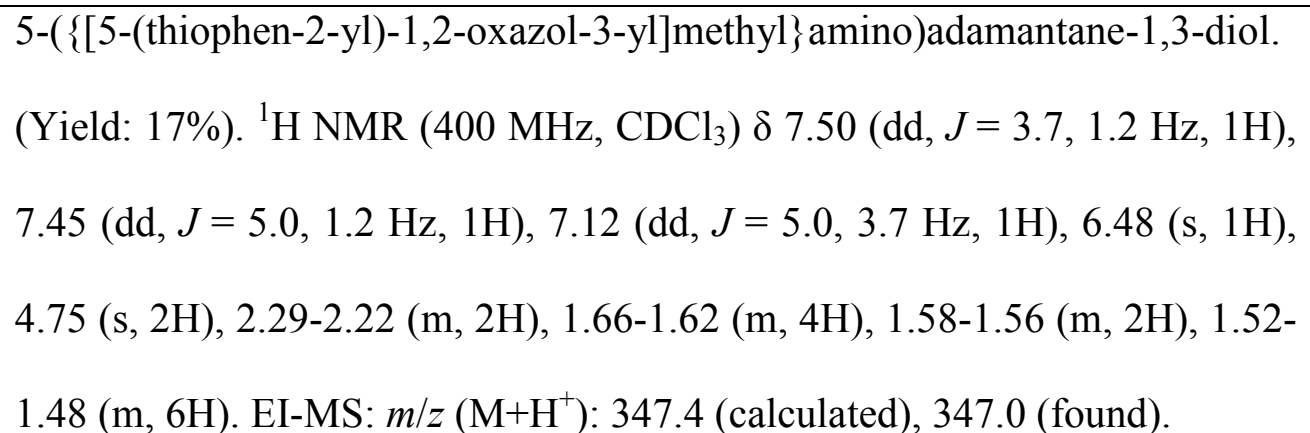 \\
\hline
\end{tabular}

\section{REFERENCES}

1. Cady, S. D.; Schmidt-Rohr, K.; Wang, J.; Soto, C. S.; DeGrado, W. F.; Hong, M. Structure of the amantadine binding site of influenza M2 proton channels in lipid bilayers. Nature 2010, 463, 689-693.

2. Choy, J.; Jaime-Figueroa, S.; Jiang, L.; Wagner, P. Synth. Commun. Novel Practical Deprotection of N-Boc Compounds Using Fluorinated Alcohols. Synth. Commun. 2008, $38,3840-3853$.

3. Smith, A. B.; Fukui, M.; Vaccaro, H. A.; Empfield, J. R. Phyllanthoside-P hyllanthostatin Synthetic Studies. 7. Total Synthesis of (+)-Phyllanthocin and (+)-Phyllanthocindiol. $J$. Am. Chem. Soc. 1991, 113, 2071-2092.

4. Wang, J.; Fan, X.; Cristian, L. (Influmedix, Inc. USA) Antiviral compounds and their methods of use. US 20120295886 Nov 22, 2012

5. Dart, M. j.; Carroll, W. A.; Florjancic, A. S.; Frost, J. M.; Gallagher, M. E.; Kolasa, T.; Li, T.; Nelson, D. W.; Patel, M. V.; Peddi, S.; Perez-Medrano, A.; Ryther, K. B.; Tietje, K. R. Compounds as cannabinoid receptor ligands and uses thereof. WO 2007140439 Dec 6, 2007 